\title{
Learning What Is Irrelevant or Relevant: Expectations Facilitate Distractor Inhibition and Target Facilitation through Distinct Neural Mechanisms
}

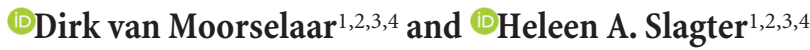 \\ ${ }^{1}$ Department of Psychology, ${ }^{2}$ Amsterdam Brain and Cognition, University of Amsterdam, 1001 NK Amsterdam, The Netherlands, ${ }^{3}$ Department of \\ Experimental and Applied Psychology, Vrije Universiteit Amsterdam, 1081BT Amsterdam, The Netherlands, and ${ }^{4}$ Institute of Brain and Behaviour \\ Amsterdam, 1081BT Amsterdam, The Netherlands
}

It is well known that attention can facilitate performance by top-down biasing processing of task-relevant information in advance. Recent findings from behavioral studies suggest that distractor inhibition is not under similar direct control but strongly dependent on expectations derived from previous experience. Yet, how expectations about distracting information influence distractor inhibition at the neural level remains unclear. The current study addressed this outstanding question in three experiments in which search displays with repeating distractor or target locations across trials allowed human observers (male and female) to learn which location to selectively suppress or boost. Behavioral findings demonstrated that both distractor and target location learning resulted in more efficient search, as indexed by faster response times. Crucially, distractor learning benefits were observed without target location foreknowledge, unaffected by the number of possible target locations, and could not be explained by priming alone. To determine how distractor location expectations facilitated performance, we applied a spatial encoding model to EEG data to reconstruct activity in neural populations tuned to distractor or target locations. Target location learning increased neural tuning to target locations in advance, indicative of preparatory biasing. This sensitivity increased after target presentation. By contrast, distractor expectations did not change preparatory spatial tuning. Instead, distractor expectations reduced distractor-specific processing, as reflected in the disappearance of the Pd event-related potential component, a neural marker of distractor inhibition, and decreased decoding accuracy. These findings suggest that the brain may no longer process expected distractors as distractors, once it has learned they can safely be ignored.

Key words: attention; brain; EEG; expectation; inhibition; statistical learning

\section{Significance Statement}

We constantly try hard to ignore conspicuous events that distract us from our current goals. Surprisingly, and in contrast to dominant attention theories, ignoring distracting, but irrelevant, events does not seem to be as flexible as is focusing our attention on those same aspects. Instead, distractor suppression appears to strongly rely on learned, context-dependent expectations. Here, we investigated how learning about upcoming distractors changes distractor processing and directly contrasted the underlying neural dynamics to target learning. We show that, while target learning enhanced anticipatory sensory tuning, distractor learning only modulated reactive suppressive processing. These results suggest that expected distractors may no longer be considered distractors by the brain once it has learned that they can safely be ignored.

\section{Introduction}

Attention can top-down bias sensory processing of task-relevant information in advance (Battistoni et al., 2017). Yet, it is currently

Received March 14, 2019; revised May 28, 2019; accepted June 6, 2019.

Author contributions: D.v.M. and H.A.S. designed research; D.v.M. performed research; D.v.M. contributed unpublished reagents/analytic tools; D.v.M. analyzed data; D.v.M. wrote the first draft of the paper; D.v.M. and H.A.S. edited the paper; D.v.M. and H.A.S. wrote the paper.

This work was supported by European Research Council Starting Grant 679399 to H.A.S. We thank Johannes Fahrenfort and Joshua Foster for valuable comments and assistance in data analysis; and Nathan Coppe for assistance in EEG data collection. debated whether distractor inhibition is under similar direct control. That is, in contrast to the widely accepted view that alpha band oscillations implement top-down inhibition (Jensen and Mazaheri, 2010; Foxe and Snyder, 2011), recent behavioral findings suggest that distractor inhibition strongly relies on previous

\footnotetext{
The authors declare no competing financial interests.

Correspondence should be addressed to Dirk van Moorselaar at dirkvanmoorselaar@gmail.com.

https://doi.org/10.1523/JNEUROSCI.0593-19.2019
}

Copyright $\odot 2019$ the authors 
experiences with visual distractions (Noonan et al., 2016; Wang and Theeuwes, 2018a). This has led to the proposal that distractor filtering is not resolved through top-down inhibition, but instead through expectation-dependent suppression of distractor processing. In this view, consistent with predictive processing theories, any expected stimulus, whether relevant or irrelevant, is suppressed (explained "away"), unless attention releases it from expectation-dependent suppression (Noonan et al., 2018).

Behavioral studies have established that (implicit) learning, where targets or distractors are most probable, influences visual selection (Geng and Behrmann, 2002, 2005; Ferrante et al., 2018; Jiang, 2018; Wang and Theeuwes, 2018b). Spatial probability learning arguably generates expectations where stimuli are most likely to occur, which may in turn bias attention. Predictive processing theories postulate that such expectations, which need not necessarily be conscious, attenuate sensory responses (Rao, 2005; Friston, 2009). Yet, ideas differ whether expectations exert their influence already in advance (Fiser et al., 2016) or, alternatively, only become apparent after stimulus presentation (Rao and Ballard, 1999; Bar et al., 2006). In line with the former notion, a recent study demonstrated expectation-dependent anticipatory sharpening of stimulus representations (Kok et al., 2017). In addition to expectation, preparatory attention also induces stimulus templates in sensory cortex that facilitate target selection (Myers et al., 2015). Expectation and attention also interact, such that top-down biasing, as reflected in prestimulus alpha lateralization, is most pronounced when targets also most likely occur at the cued, task-relevant location (Alilović et al., 2019). Thus, taskrelevant expectations may bias corresponding visual regions in advance to facilitate goal-directed behavior. Yet, how distractorspecific expectations help resolve interference, through modulating prestimulus activity representing the distracting information and/or postdistractor processing, is unclear.

Notably, a recent study reported no changes in prestimulus alpha lateralization as a function of distractor location learning despite clear behavioral evidence for distractor suppression (Noonan et al., 2016). This absence of any distractor-related alpha lateralization is surprising in light of the prevailing view that alpha oscillations implement top-down inhibition of activity in irrelevant visual networks (Jensen and Mazaheri, 2010; Foxe and Snyder, 2011). Yet, the vast majority of this work examined inhibition in binary designs, where one of two features was irrelevant. Hence, observed effects could also reflect secondary inhibition related to more attention to task-relevant features, or simply attending away, rather than top-down inhibition per se (Foster and Awh, 2018).

The present study was designed to characterize the neural mechanisms underlying learned distractor suppression and directly contrast these with target learning. Specifically, we aimed to establish whether learned suppression is purely reactive or already evident in anticipation of expected distractors. For this purpose, in two behavioral and one EEG experiment, across visual searches, either the distractor or the target location was repeated, allowing observers to learn which location to selectively ignore or select (see Fig. 1A). As target locations could not be predicted in the distractor-repeat condition, any observed effect of distractor learning is unlikely to be driven by more attention to expected target locations. Changes in preparatory and stimulusinduced activity and neural representation were examined using inverted encoding modeling, event-related potential (ERP), and multivariate pattern analyses. Based on recent studies showing expectation- and attention-dependent anticipatory sharpening of stimulus representations (Samaha et al., 2016; Foster et al.,
2017), we expected target learning to enhance reconstruction of target locations before search display onset. However, if and how distractor learning also changed spatial distractor tuning in advance or only modulated postdistractor processing was an open question.

\section{Experiment 1: the time course of distractor learning}

To study the neural mechanisms underlying learned distractor suppression, it is important to first characterize the rate at which suppression develops. We therefore first ran two behavioral experiments in which observers performed sequences of 12 visual searches with fixed distractor locations (see Fig. 1A). In Experiment 1, in addition, we included a baseline condition in which the target and the distractor location varied from trial to trial. The comparison between distractor-repeat and baseline sequences allowed us to assess the minimum number of distractor repetitions necessary to observe reliable suppression effects. In a second control condition, only a target was presented, the location of which varied over trials within a sequence to assess whether learned suppression can completely counteract distractor interference, as has been observed in additional singleton paradigms (Gaspelin et al., 2015; Gaspelin and Luck, 2018a) and, if so, how quickly. Critically, in this target-only variable condition, one of the placeholders was eliminated. In combination with a set size modulation (i.e., each sequence contained four or eight placeholders), this manipulation allowed us to exclude the possibility that the hypothesized suppressive effects in distractor-repeat sequences simply reflect facilitated processing at locations that are known to never contain a distractor. If so, distractor-repeat and target-variable conditions should demonstrate highly similar set size modulations.

\section{Materials and Methods}

Experiment and analyses were based on the AsPredicted registration at Open Science Framework (https://osf.io/t9qxu/).

Participants. A planned number of 18 participants (mean age $=24$ years, range 19-34 years; 7 men) participated in Experiment 1, in exchange for course credit or monetary compensation (10 euros per hour). Three participants were replaced, all because overall error rates were $>2.5$ SDs below group average. Participants reported normal or corrected-to-normal vision and provided written informed consent before participation. The ethical committee of the Department of Psychology of the University of Amsterdam approved the study.

Apparatus, stimuli, design, and procedure. A Windows 7 PC running OpenSesame version 3 (Mathôt et al., 2012) using PsychoPy (Peirce, 2009) functionality generated the stimuli on an ASUS VG236 $120 \mathrm{~Hz}$ monitor with a gray background, at $\sim 80 \mathrm{~cm}$ viewing distance. Participants sat in a dimly lit room.

Each trial started with a $500 \mathrm{~ms}$ black fixation dot with a white rim (radius 5 pixels) followed by a placeholder display. This display contained four or eight black rimmed placeholders (radius 75 pixels), all placed on an imaginary circle centered (radius 225 pixels) on fixation. After a $250 \mathrm{~ms}$ delay, two Gabor patches $(\mathrm{sf}=0.025$, contrast $=1$ ) were presented for $200 \mathrm{~ms}$ within the centers of two selected placeholders. One Gabor, the target, was tilted left or right $\left(45^{\circ}\right.$ and $\left.135^{\circ}\right)$, while the other Gabor, the distractor, was randomly vertically or horizontally oriented. Participants had to indicate the orientation of the target Gabor (left or right) via keyboard response. They were instructed to respond as fast as possible while trying to keep the number of errors to a minimum. A trial ended when participants made a response via a keyboard press or 1000 ms after search display onset.

Crucially, there were three separate conditions. In the distractorrepeat condition, the location of the distractor was fixed across a sequence of 12 trials, while the location of the target was selected semirandomly (i.e., the target location was never repeated on consecutive trials). In the baseline condition, target and distractor locations were random, with the restriction that neither the target nor distractor location repeated on consecutive trials. In the target-only variable condition, the target was not accompanied by a distractor and its location was selected at random, again with the restriction that it did not repeat on consecutive trials. Also, in this condition, one of the placeholders was 
removed, in effect reducing the number of potential target locations by one relative to baseline and distractor-repeat conditions to establish whether repetition benefits in distractor-repeat sequences could be explained by shifts of attention away from the distractor location. At the start of each 12-trial sequence, the fixation point turned red for $50 \mathrm{~ms}$. Search display set sizes were fixed within repetition sequences.

Participants completed 36 practice trials in which only the distractor location was repeated, and 27 experimental blocks ( 9 blocks for each condition) of 72 trials each, with condition order counterbalanced over participants. Each experimental block contained three set size 4 and three set size 8 repetition sequences, randomly intermixed, resulting in 27 trials per trial position in the sequence for each condition. At the start of each block, participants were informed about the upcoming condition. Participants were encouraged to take a break between blocks.

Analysis. All data were analyzed in a Python environment (Python Software Foundation, https://www.python.org/). As preregistered, behavioral analyses were limited to reaction time (RT) data of correct trials only. RTs were filtered in a two-step trimming procedure: trials with RTs $<200$ ms were excluded, after which data were trimmed on the basis of a cutoff value of $2.5 \mathrm{SD}$ from the mean per participant per condition. Remaining RTs were analyzed with repeated-measures ANOVAs followed by planned comparisons with paired $t$ tests using JASP software (JASP-TEAM, 2018).

\section{Results and Discussion}

Search times

Exclusion of incorrect responses $(11.3 \%)$ and data trimming $(1.8 \%)$ resulted in an overall loss of $13.1 \%$ of the data. Remaining RTs were entered into a repeated-measures ANOVA with the within-subject factors condition (distractor-repeat, baseline, target-only variable), set size $(4,8)$, and trial position (1-12). As visualized in Figure $1 B$, the main effect of condition $\left(F_{(2,34)}=\right.$ 99.1, $\left.p<0.001, \eta^{2}=0.85\right)$ was accompanied by decreasing RTs over trials $\left(F_{(11,187)}=56.8, p<0.001, \eta^{2}=0.77\right)$, and overall faster RTs at set size 4 than at set size $8\left(F_{(1,17)}=20.5, p<0.001\right.$, $\left.\eta^{2}=0.55\right)$. An interaction between condition and trial position showed that, as expected, decreasing RTs across trials was largely specific to distractor-repeat sequences $\left(F_{(22,374)}=12.1, p<0.001\right.$, $\left.\eta^{2}=0.42\right)$, the only condition in which location expectations could develop. The overall slower response in the first compared with the second trial observed in all conditions, we attribute to the start of a new sequence.

Planned comparisons between baseline and distractor-repeat sequences demonstrated that a single repetition was sufficient to observe reliable benefits both at set size $4\left(t_{(17)}=3.0, p=0.008\right)$ and set size $8\left(t_{(17)}=4.8, p<0.001\right)$, and this benefit of distractor foreknowledge continued to increase across repetitions (all $t$ values $>3.2$, all $p$ values $<0.006$ ). However, distractor learning never completely counteracted distractor interference, as at the end of the sequence, RTs only numerically approached the targetonly variable condition but were still reliably slower $\left(t_{(17)}=3.7\right.$, $p=0.002$ for set size $4 ; t_{(17)}=5.6, p<0.001$ for set size 8 ).

A nonsignificant set size $\times$ trial position interaction suggested that the rate at which distractor learning developed was independent from the number of placeholders $(F=1.3, p=0.22)$. Yet, as also visualized in Figure $1 B$ and confirmed by a three-way interaction $\left(F_{(22,374)}=1.6, p=0.041, \eta^{2}=0.09\right)$, distractor-repeat sequences did yield a significant set size $\times$ trial position interaction $\left(F_{(11,187)}=64.9, p<0.001, \eta^{2}=0.79\right)$, which would also be expected if the repeated location was not suppressed, but observers instead shifted their attention to the remaining locations. To address this issue, we normalized the data relative to the first trial and fitted each individual's data to an exponential decay function: $D+(1-D){ }^{*} \alpha^{\text {rep}}$, where $D$ equals the absolute reduction relative to the first trial and $\alpha$ is rate at which this reduction develops. This analysis revealed that the set size modulation was evident in the reduction boundary $\left(t_{(17)}=4.3, p<0.001\right)$, but not in the learning rate $(t=0.2, p=0.88)$. No such effects were observed in baseline or, importantly, target-only variable conditions (all $t$ values $<1.19$, all $p$ values $>0.3$ ), indicating that the observed benefits in distractor-repeat sequences reflect learned location suppression, rather than increased attention toward the remaining possible target locations.

To summarize, the results from Experiment 1 demonstrate that learning about the spatial probability of a distractor increases search efficiency. Distractor-based learning was implemented relatively quickly, although after 11 repetitions, distractor interference was not yet fully resolved, as RT was not yet as fast as in the target-only variable condition.

\section{Experiment 2: the time course of learned suppression and learned facilitation}

Experiment 1 showed that 11 distractor location repetitions increasingly speeded up RTs, indicative of distractor learning. Although this effect differed between set sizes, it did not seem mediated by more attention to potential target locations (or a reduction in target location uncertainty), as the set size modulation was absent in target-only variable sequences and did not modulate learning rate in distractor-repeat sequences. Experiment 2 was designed to replicate the effect of distractor learning on performance observed in Experiment 1, and directly contrast this to the time course of target learning.

\section{Materials and Methods}

The methods and analyses were preregistered (https://osf.io/t8c4m/) and were the same as in Experiment 1, except for the following changes: A planned number of 20 new participants (mean age $=23$ years, range 18-27 years; 7 men) participated. Two participants were replaced: 1 because overall RTs were $>2.5$ SDs above group average and 1 because overall error rates were $>2.5$ SDs below group average.

In Experiment 2, there were two conditions. Next to the distractorrepeat condition (used in Experiment 1), here we also included a targetrepeat condition, in which the location of the target was repeated over trials, while the location of the distractor was selected semirandomly (i.e., the distractor location was never repeated on consecutive trials). All participants completed 18 experimental blocks, with repetition condition blocked in counterbalanced order.

\section{Results and Discussion}

Exclusion of incorrect responses (10.8\%) and data trimming $(2.3 \%)$ resulted in an overall loss of $12.9 \%$ of the data. As visualized in Figure $1 C$, RTs again decreased across trials $\left(F_{(11,209)}=\right.$ 374.3, $\left.p<0.001, \eta^{2}=0.95\right)$, with overall faster RTs in target than distractor repetition sequences $\left(F_{(1,19)}=106.4, p<0.001, \eta^{2}=\right.$ $0.85)$ and for set size 4 compared with set size 8 displays $\left(F_{(1,19)}=\right.$ 34.0, $\left.p<0.001, \eta^{2}=0.64\right)$. Significant interactions demonstrated that the repetition benefit was more pronounced when the target location was repeated (interaction between condition and trial position; $\left.F_{(11,209)}=24.3, p<0.001, \eta^{2}=0.56\right)$ and when there were only four placeholders (interaction between set size and trial position; $\left.F_{(11,209)}=2.2, p=0.017, \eta^{2}=0.10\right)$. Critically, the effect of distractor repetition on RT was not differently affected by set size than the effect of target repetition on RT, as the three-way interaction between condition, trial position, and set size was not significant $(F=1.6, p=0.11)$. Separate ANOVAs on the parameters resulting from the same model fits as in Experiment 1 yielded no significant condition $\times$ set size interactions, on either learning rate or on reduction boundary (all $F$ values $<1.9$, all $p$ values $>0.18$ ), further arguing against the notion that, in distractor-repeat blocks, observers simply paid more attention to potential target locations. 


\section{A}

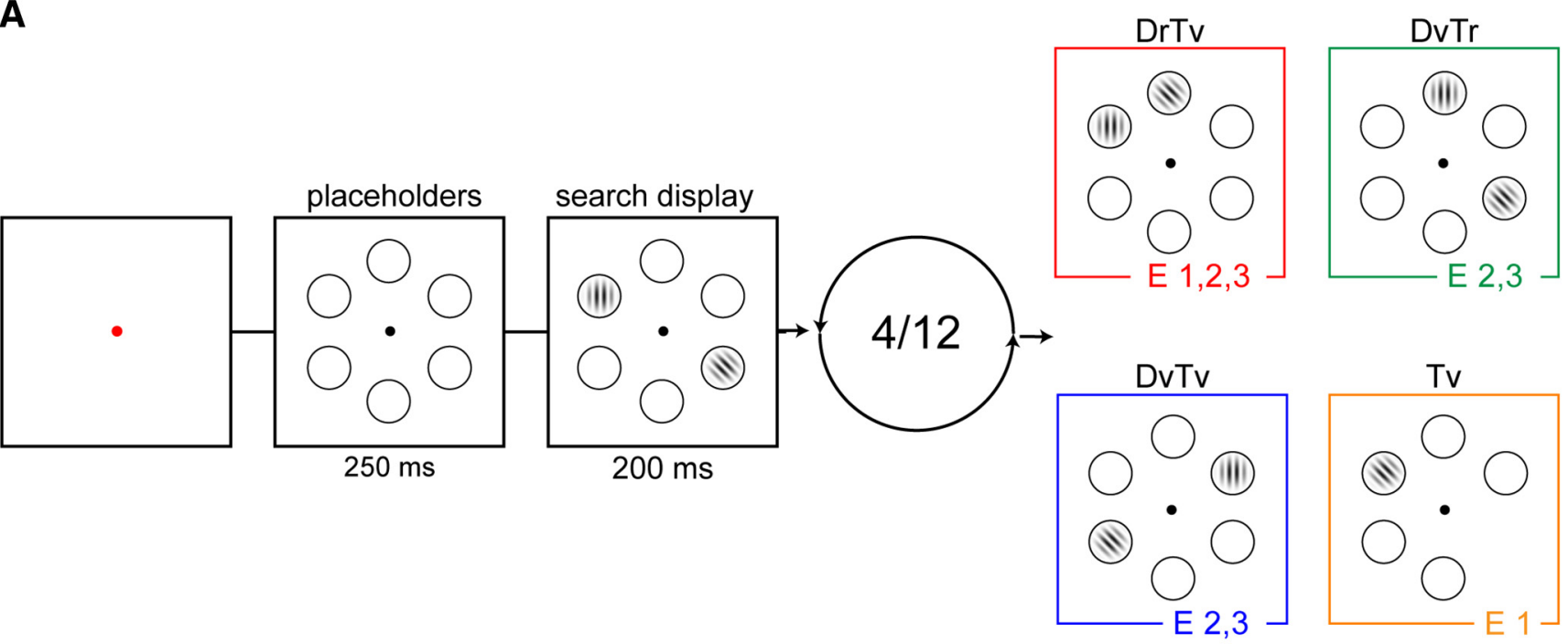

B

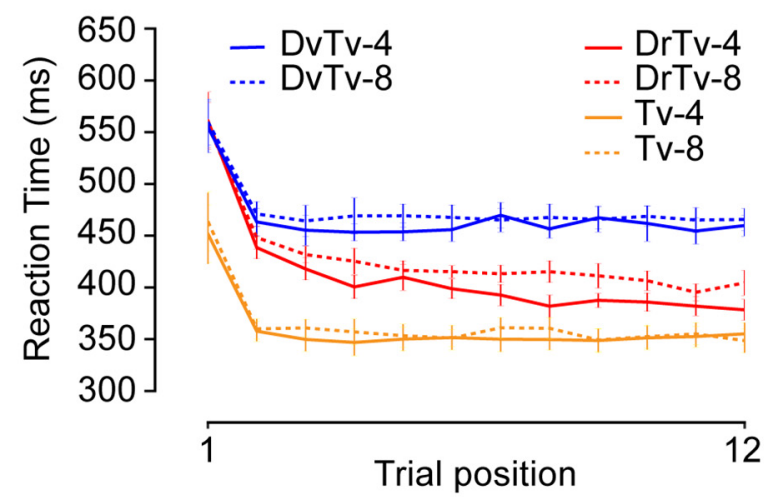

D

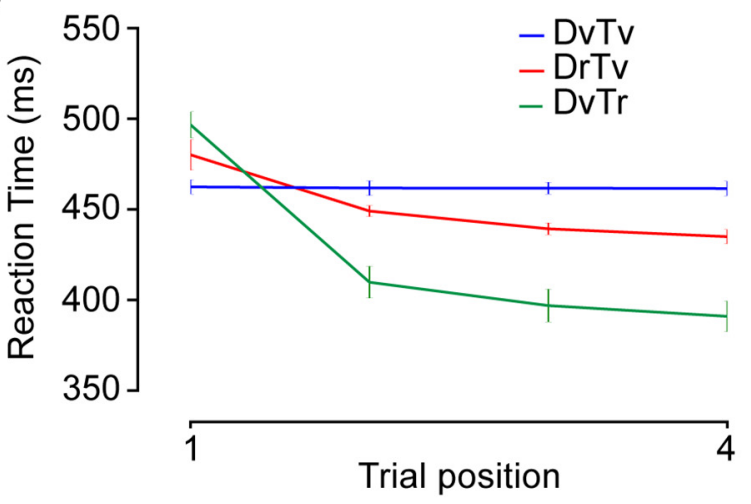

C

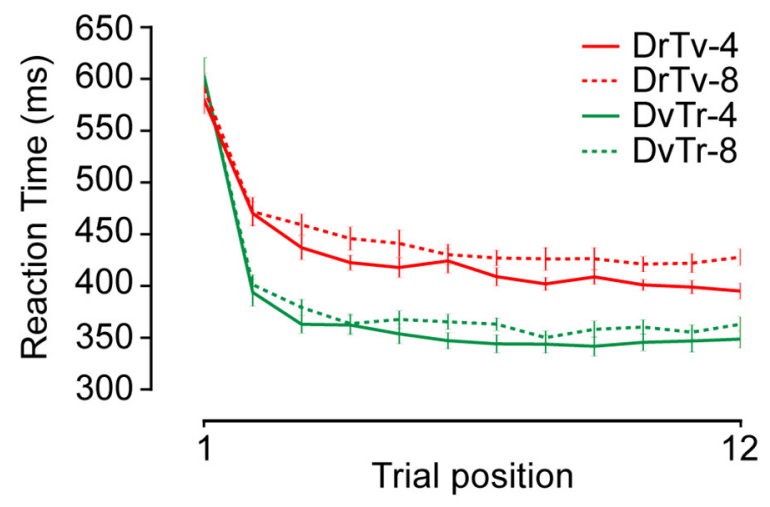

$E$

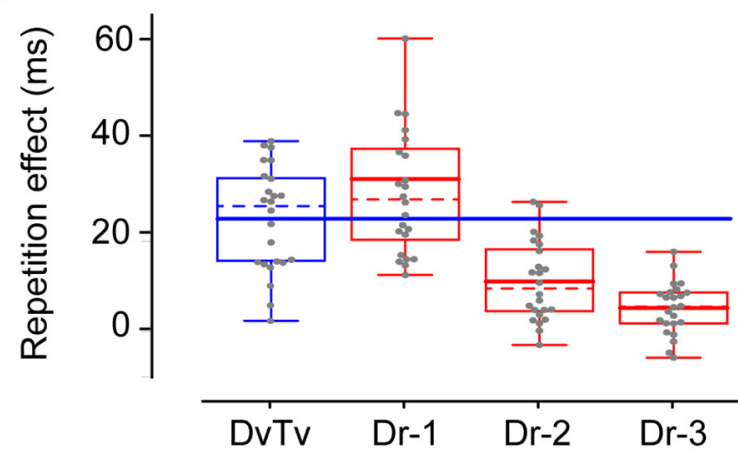

Figure 1. Task design and behavioral findings of Experiments 1-3. A, A trial sequence of Experiments 1-3. In each condition, in each trial of a sequence of trials, participants had to indicate the orientation (left or right) of a target Gabor. In all conditions except one, a distractor (a Gabor that was horizontally or vertically oriented) was concurrently presented. Across a sequence of trials, the distractor location could repeat, the target location could repeat, or target and distractor locations varied across trials. The search display was presented for 200 $\mathrm{ms}$, and participants had $1000 \mathrm{~ms}$ to respond. In all conditions, except the target-only variable (Tv) condition, the target was accompanied by a distractor (a horizontally or vertically oriented Gabor). In the target-repeat (DvTr) and distractor-repeat (DrTv) conditions, the location of the target (Tr) or the distractor (Dr) was repeated over trials in a sequence. In the baseline (DvTv) condition, the target and distractor location varied from trial to trial. In the target-only variable condition (Tv), the location of the target also varied from trial to trial. The number of trials in a sequence ranged between 4 and 12 , and the number of search locations between 4 and 8 across experiments. Further, the colors of each condition correspond to condition specific colors in subsequent plots. $\boldsymbol{B}-\boldsymbol{D}$, RTs as a function of condition and trial position for $(\boldsymbol{B})$ Experiment $1,(\boldsymbol{C})$ Experiment 2, and (D) Experiment 3. $\boldsymbol{E}$, Boxplot showing benefits of distractor location repetition in distractor-repeat sequences in Experiment 3 as a function of trial position contrasted to random distractor location repetitions in baseline sequences. Solid lines inside boxes indicate the mean. Dashed lines indicate the median. 
In summary, the results of Experiment 2 again demonstrate that distractor-based learning is implemented relatively quickly, although not as rapidly as target-based learning. Together, these experiments demonstrate that, although a single repetition of the distractor already facilitates performance, the benefit of learning the spatial probability of a distractor increases gradually over trials. Next, we aimed to assess the neural mechanisms that underlie distractor learning-related behavioral benefits and contrast these to the neural mechanisms underlying target location learning: How does experience with distracting, but irrelevant information, help the brain resolve distractor interference?

\section{Experiment 3: neural mechanisms underlying learned facilitation and inhibition}

Experiment 3 was designed to investigate the neural mechanisms that underlie the behavioral effects of distractor and target learning observed in the previous experiments. We were specifically interested to establish whether learning about the spatial probability of a distractor, just like learning about the spatial probability of a target, changes anticipatory tuning to the expected distractor location, reflective of preparatory inhibition, and/or suppresses distractor-related processing. To test this, we measured EEG and contrasted neural dynamics of learned suppression and facilitation across repetitions. We designed the experiment such that we could fit an inverted encoding model (IEM) to reconstruct both the target and the distractor location from the multivariate EEG activity. This required that each location in the display was repeated equally often to prevent systematic biases in the model fits. Consequently, we had to reduce the sequence length to four repetitions, which, based on our Experiments 1 and 2, was sufficient to observe robust behavioral benefits of distractor learning.

In addition, here, we aimed to parse out the influence of immediate distractor repetitions within the context of learned suppression. Based on the first two experiments, it remains unclear whether learned suppression goes above and beyond intertrial priming. Previous studies have already shown that distractor repetition-related reductions in RT remain reliable when controlling for intertrial priming effects in search tasks in which the distractor was more likely to occur at one location that remained the same across the entire experiment (Wang and Theeuwes, 2018b; Failing et al., 2019). Yet, this control still allows for influences resulting from more distant trials in the past. Here, we investigated whether expected repetitions benefit distractor processing more than unexpected, random repetitions in a much shorter learning context.

\section{Materials and Methods}

The methods and analyses (preregistered at https://osf.io/4bx7y) were identical to those from Experiments 1 and 2, except for the following changes: A planned number of 24 new participants (mean age $=23$ years, range 19-37 years; 6 men) participated in the experiment. Four participants were replaced: 2 because they did not complete all experimental sessions and 2 because preprocessing (for details, see below) of EEG data resulted in exclusion of too many trials $(>30 \%)$. Participants were seated in a dimly lit testing room, and all stimuli were presented at a distance of $\sim 70 \mathrm{~cm}$. Manual responses were collected via two purpose-built response buttons, which were positioned at the end of the armrests of the participant's chair.

In contrast to Experiments 1 and 2, there were always six search locations and sequences were reduced to four trials. There were three experimental conditions, which were presented in separate blocks. In distractor-repeat and target-repeat conditions, the distractor or target location, respectively, was repeated. The location of the other item (target or distractor, respectively) was never repeated on consecutive trials and was placed maximally twice on the same location within a four-trial sequence. In the third, baseline condition, the target and distractor location were again variable with the restriction that the target location was never repeated and the distractor could maximally be presented two times at the same location in a sequence of four trials. This latter manipulation allowed us to contrast unexpected distractor repetition in base- line sequences (i.e., distractor priming) to learned suppression in distractor-repeat sequences. The length of the fixation display preceding each trial was randomly jittered between $450 \mathrm{~ms}$ and $750 \mathrm{~ms}$, and each response was followed by a $200 \mathrm{~ms}$ blank screen.

Participants came to the laboratory twice. In each session, they completed 51 blocks of the task of 72 trials each, with condition blocked in counterbalanced order (e.g., target-repeat, baseline, distractor-repeat, etc.), while their brain activity was recorded with EEG and their eye movements were monitored with an eye tracker. This resulted in 612 observations per condition and trial position within a four-trial sequence. In the first session, participants first completed a series of 24 practice trials in which only the distractor location was repeated.

Analysis software. Preprocessing and subsequent analyses were performed using custom-written analysis scripts, which are largely based on functionalities implemented within MNE (Gramfort et al., 2014). These scripts can be downloaded at https://github.com/dvanmoorselaar/DvM.

EEG recording and preprocessing. EEG data were recorded at a sampling rate of $512 \mathrm{~Hz}$ using a 64-electrode cap with electrodes placed according to the 10-20 system (using a BioSemi ActiveTwo system; www.biosemi. com). All electrodes were rereferenced offline to the average of two channels placed at the left and right earlobes, respectively. External electrodes placed $\sim 2 \mathrm{~cm}$ above and below the right eye and $\sim 1 \mathrm{~cm}$ lateral to the external canthi were used to measure vertical EOG and horizontal EOG, respectively.

Continuous EEG of the two sessions was high-pass filtered using a zero-phase 'firwin' filter at $0.1 \mathrm{~Hz}$ as implemented in MNE to remove slow drifts, and subsequently epoched from -550 to $550 \mathrm{~ms}$ relative to the onset of the search display, extended by $500 \mathrm{~ms}$ at the start and end of the epoch to control for filter artifacts during preprocessing and timefrequency analyses. The resulting epochs were baseline normalized using the whole epoch as a baseline. Before cleaning, EEG signals were visually inspected for malfunctioning electrodes, which were excluded from subsequent preprocessing steps (mean $=2$, range $0-5$ ). To detect epochs contaminated with noise, we filtered the EEG signal with a $110-140 \mathrm{~Hz}$ bandpass filter and then used an adapted version of an automatic trialrejection procedure as implemented in the Fieldtrip toolbox (Oostenveld et al., 2011), allowing for variable $z$ score cutoffs per participants based on the within-subject variance of $z$ scores (de Vries et al., 2017). This resulted in an average rejection of $9.2 \%$ of all trials (range $2.3 \%-20.2 \%$ ). After trial rejection, independent component analysis, as implemented in MNE using the 'extended-infomax' method, was performed on nonepoched $1 \mathrm{~Hz}$ high pass-filtered data to identify and remove eye-blink components from the $0.1 \mathrm{~Hz}$ filtered data. Finally, malfunctioning electrodes were interpolated using spherical splines (Perrin et al., 1989) before the data of the separate sessions were combined.

Throughout EEG recording, eye movements were monitored using an Eyelink 1000 (SR Research), sampled at $500 \mathrm{~Hz}$. Gaze data were analyzed online, so that every time the participant broke fixation, auditory feedback was provided, signaling to the participant to keep their gaze at fixation. The Eyelink data were also analyzed offline. To control for drifts in the eye-tracker, epochs without a saccade (Nyström and Holmqvist, 2010) in the $300 \mathrm{~ms}$ predisplay interval were shifted toward fixation. Each epoch was then summarized by a single value indicating the largest deviation measured in a segment of data $(>40 \mathrm{~ms})$. The results of the EEG analyses presented next are limited to trials with a fixation deviation $<=1^{\circ}$ or, in case of missing Eyelink data, trials with sudden sharp jumps in the horizontal EOG as detected via a step algorithm $($ mean $=7.2 \%$, range $=0.3 \%-18.3 \%$ combined for eye tracker analysis and step algorithm).

Time-frequency analysis. To isolate frequency-specific activity, we bandpass filtered the raw EEG signal using a Butterworth bandpass filter (order $=5$, frequency band $=8-12$ ) within MNE ("filter_data" from MNE toolbox) (Gramfort et al., 2014). Subsequently, the filtered signal was downsampled to $128 \mathrm{~Hz}$ to reduce computational time of the IEM analysis. A Hilbert Transform (Scipy) (Oliphant, 2007) was then applied to the bandpass-filtered data to produce the complex analytic signal of the filtered EEG.

We focused our analyses both on evoked and total power. Total power was computed by squaring the complex magnitude of the complex ana- 
lytic signal and then averaged across trials. Consequently, total power reflects the ongoing activity regardless of its phase relationship to the onset of the stimulus displays. In contrast, evoked power was computed by averaging the complex analytical signal across trials before power extraction such that evoked power reflects activity phase-locked to stimulus onset because only activity with consistent phase across trials remains after averaging the complex analytic signal.

Because calculating evoked power requires averaging across trials, following previous work, artifact-free trials were partitioned into three sets: two training and one testing set (see IEM). In doing so, we equated the number of observations across stimulus locations and conditions to prevent bias in the analysis. To this end, we calculated the minimum number of trials $n$ for each location for each participant and assigned $n / 3$ many trials for each location to each of the three sets while ensuring that no trial was repeated across blocks (i.e., sets were independent). Consequently, a random subset of epochs was not included in data partitioning. To account for this, results were averaged across 10 data divisions, in which each of three datasets served as a testing set once.

IEM. To determine effects of distractor and target learning on (preparatory) spatial tuning of population-level activity, we applied an IEM (Brouwer and Heeger, 2009) to the topographic distribution of alpha power across electrodes to create location-selective channel tuning functions (CTFs). This critically allowed us to examine effects of distractor and target learning on spatial tuning of population-level activity. The IEM procedure reverses the direction of inference from a decoding analysis by capturing the continuous relationship between stimulus position and multivariate EEG. Following Brouwer and Heeger (2009), we used the same procedure reported in previous work (van Moorselaar et al., 2018) to separately reconstruct the target and the distractor location on the first and final trial across conditions.

Here, we modeled the hypothetical response in each of the six stimulus position channels (i.e., neuronal population) as half sinusoid raised to the seventh power and centered on the polar angle of each corresponding spatial channel. The resulting six basis sets were used to construct a $k \times n$ response prediction matrix $C_{1}$, where $k$ is the number of position channels (i.e., six) and $n$ is the number of observations in the training set. An IEM routine was then applied that proceeded in two phases. In the training phase, the mapping from "channel" to "electrode" space was estimated by performing an ordinary least-squares regression of the $C_{1}$ matrix onto the $m \times n_{1}$ observed power train matrix $B_{1}$, where $m$ is the number of electrodes (i.e., 64) and $n_{1}$ is the number of observations in the training set. This regression yields an $m \times k$ weight matrix $W$, where each electrode in $m$ contains a regressor weight for each spatial channel in $k$. Next, in the test phase, the model was inverted by performing ordinary least squares again, but now regressing these weights onto the $m \times n_{2}$ observed power test matrix $\mathrm{B}_{2}$, where $m$ is the number of electrodes and is the number of observations in the testing set. This regression transforms the test data $B_{2}$ into an $k \times n_{2}$ estimated channels responses matrix $C_{2}$, where each spatial channel $k$ contains an estimated response for all observations in $n_{2}$. Finally, each estimated CTF was circularly shifted to a common center. This routine was performed separately for each sample point from $-550 \mathrm{~ms}$ before search display onset until $550 \mathrm{~ms}$ postsearch display onset.

Importantly, we used a leave-one-out cross-validation routine, wherein two sets serve as $B_{1}$ to estimate $W$, and the remaining block served as $B_{2}$ and was thus used to estimate $C_{2}$. This routine was repeated until each set had served as testing set (i.e., $B_{2}$ ).

To evaluate reconstruction of the target and distractor location, we estimated CTF slopes. For this purpose, we collapsed across channels that were equidistant from the center and used linear regression to calculate the slope of the CTF, where a positive slope indicates greater location selectivity (relative to the other locations), a flat slope no location specificity, and a negative slope reduced location selectivity (relative to the other locations). Previous work using IEMs has demonstrated that the reconstruction of spatially selective CTFs during preparatory spatial attention is largely specific to power in the alpha band (Samaha et al., 2016; Foster et al., 2017; van Moorselaar et al., 2018). To confirm that reconstruction was most pronounced within the alpha band, we also searched a broad range of frequencies $(4-30 \mathrm{~Hz}$, in increments of $2 \mathrm{~Hz}$ with a $4 \mathrm{~Hz}$ band; $4-8 \mathrm{~Hz}, 6-10 \mathrm{~Hz}$, etc.) using the collapsed data of all conditions of interest.

ERP analysis. We next examined how learning changed distractor and target processing after stimulus presentation using ERPs. To enable isolation of target- and distractor-specific ERP components, the primary analysis focused on trials in which the stimulus of interest (target or distractor) was presented to the left or right of fixation below the horizontal midline, whereas the other stimulus was presented on the vertical meridian. Limiting the analyses to these stimulus configurations enabled isolation of the lateralized ERP components, the N2pc and the Pd, because vertical midline singletons do not evoke such lateralized activity (Woodman and Luck, 2003; Hickey et al., 2009). Waveforms to the various search displays were collapsed across left and right visual hemifield and left and right electrodes to produce separate waveforms for contralateral and ipsilateral scalp regions. Lateralized difference waveforms relative to the target and the distractors were then computed by subtracting the ipsilateral waveform from the corresponding contralateral waveform.

After cleaning, epochs were $30 \mathrm{~Hz}$ low-pass filtered and baseline corrected using a -300 to $0 \mathrm{~ms}$ preplaceholder onset baseline period. ERP averages were balanced to contain an equal number of observations across conditions and trial position. We were primarily interested in ERP components related to visual processing and attention, namely, the $\mathrm{P} 1$, $\mathrm{N} 1, \mathrm{~N} 2 \mathrm{pc}$, and the Pd, which are typically observed at lateral posterior electrodes sites. Based on visual inspection of the topographic distribution of condition-averaged voltage values in these regions, we selected $\mathrm{O} 1 / \mathrm{O} 2, \mathrm{PO} 3 / \mathrm{PO} 4$, and $\mathrm{PO} 7 / \mathrm{PO} 8$ as our electrodes of interest.

$\mathrm{P} 1$ and $\mathrm{N} 1$ windows, $110-150 \mathrm{~ms}$ and $160-220 \mathrm{~ms}$, respectively, were selected based on visual inspection of the group and condition-averaged waveforms. For each subject, we obtained the mean voltage value over a $35 \mathrm{~ms}$ time window centered around the $\mathrm{P} 1$ or N1 peaks within these selected time windows. N2pc and Pd windows, 140-200 ms and 280-360 $\mathrm{ms}$, respectively, were also selected via visual inspection of the group and condition-averaged waveforms, but now using contralateral-ipsilateral difference waveforms.

Multivariate decoding analyses. Finally, we also applied multivariate decoding analysis to decode target and distractor locations. This approach allowed us to examine whether the neural signal contained location-specific information that may not have been captured by the assumptions in our IEM. Also, this approach provides a more finegrained spatial profile of attentional selection than lateralized ERP components (Fahrenfort et al., 2017), which by definition are driven by activation differences between cortical hemispheres.

To decrease the computational time of the decoding analysis, the EEG signal was downsampled to $128 \mathrm{~Hz}$. We applied a linear classification algorithm (Pedregosa et al., 2011) in electrode space (64 electrodes) at each time sample, using a 10-fold cross-validation scheme. Decoding was done for the first and final trial in a repetition sequence in each condition using single trial data. We entered the six stimulus locations into the classifier as six different stimulus classes while ensuring that each stimulus class was selected equally often. This analysis was run separately for distractor and target locations. The classifier was trained on $90 \%$ of the data and tested on the remaining $10 \%$ of the data, a procedure that was repeated until all data were tested exactly once. Classification accuracy was computed as the percentage of correct class assignments averaged across the 10 folds.

Statistics. To evaluate how location repetition changed the neural representation of target and distractor locations, we contrasted target-repeat sequences and distractor-repeat sequences with baseline sequences using group-level permutation testing with cluster correction for multiple comparisons (Maris and Oostenveld, 2007). First, for the first and final trial within a sequence, the conditions of interests were contrasted to the baseline condition (e.g., target-repeat 4 vs baseline 4 ). Second, significant clusters on the final trial were evaluated again after controlling for potential differences between repeat and baseline sequences at the start of the sequence (e.g., target repeat 4 - baseline 4 vs target repeat 1 baseline 1).

In addition to cluster-based, group-level permutation testing, specific comparisons were also evaluated using repeated-measures ANOVAs. 
A

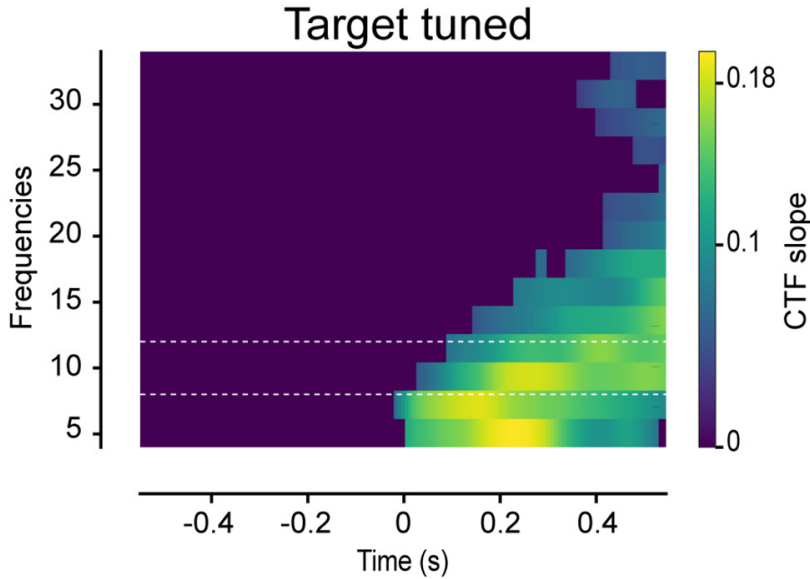

B

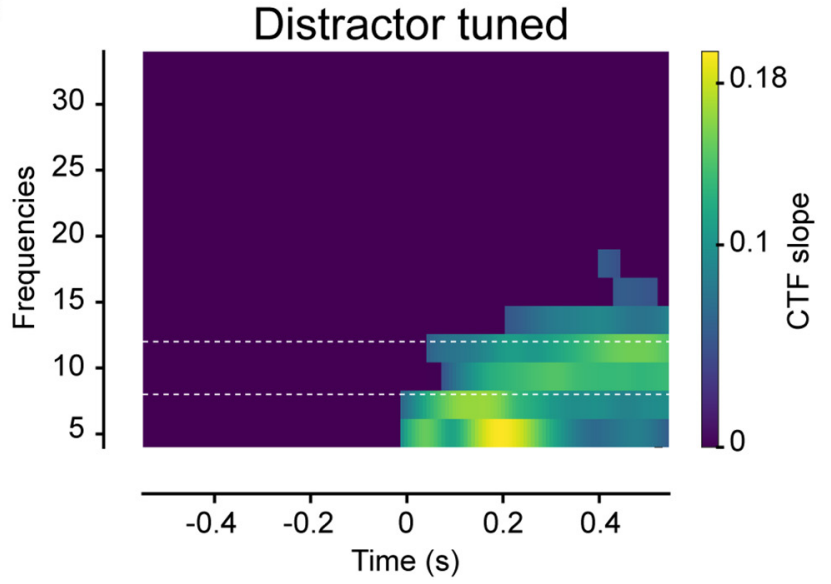

Figure 2. Topographic power in a range of low frequencies tracks both the location of the target and the distractor. $\boldsymbol{A}$, Total power CTF slopes tuned to the target location across a range of frequencies and collapsed across all conditions of interest (i.e., DvTv1, DvTv4, DvTr1, DvTr4). All nonsignificant values were set to zero in a tw0-step procedure. First, each individual data point was tested against zero with a paired-sampled $t$ test. After setting nonsignificant values to zero, data were evaluated using cluster-based permutation. $\boldsymbol{B}$, Total power CTF slopes tuned to the distractor location across a range of frequencies and collapsed across all conditions of interest (i.e., DvTv1, DvTv4, DrTv1, DrTv4).

While permutation testing has the merit of not choosing specific time points a priori and therefore allows to observe potentially nonexpected effects, it also has the risk of missing brief, but reliable, effects. Moreover, as tests are conducted per sample, they are more sensitive to noise than analyses conducted on measures that are obtained by averaging across several samples. Therefore, when we expected ERP effects within specific time windows, we also evaluated their presence using repeated-measures ANOVAs, as is common in ERP research. These tests were also used in case only one of the two permutation contrasts described above identified a significant cluster.

\section{Results}

Search times

Exclusion of incorrect responses (7.2\%) and data trimming (2.4\%) resulted in an overall loss of $9.7 \%$ of the data. Figure $1 D$ illustrates that RTs decreased with repetition (main effect trial position; $F_{(3,69)}=105.1, p<0.001, \eta^{2}=0.82$ ), with overall fastest RTs following target repetition and slowest RTs without repetition (main effect condition; $F_{(2,46)}=60.8, p<0.001, \eta^{2}=$ 0.73 ). While repetition benefits were most pronounced in targetrepeat blocks (interaction effect condition and trial position; $\left.F_{(6,138)}=101.2, p<0.001, \eta^{2}=0.82\right)$, distractor repetition also speeded search: A separate ANOVA without target-repeat blocks also yielded a highly significant interaction effect $\left(F_{(3,69)}=54.5, p\right.$ $\left.<0.001, \eta^{2}=0.70\right)$. Planned pairwise comparisons demonstrated that, within both target and distractor repeat sequences, each subsequent repetition speeded RTs (all $t$ values $>4.0$, all $p$ values $<0.001$ ), whereas no such effect was observed in the baseline condition in which targets and distractor occurred at different locations from trial-to-trial and no expectations could develop (all $t$ values $<0.4$, all $p$ values $>0.7$ ). These observations show that both benefits of target and distractor learning developed gradually across repetitions. Importantly, as visualized in Figure $1 E$, the first repetition in distractor-repeat sequences speeded search more than random repetitions in baseline sequences $\left(t_{(23)}=2.5, p=0.021\right)$. This finding indicates that, although a part of our behavioral effects was likely driven by intertrial priming, learned biases are additive to these pure repetition effects, in line with previous reports (Wang and Theeuwes, 2018b; Failing et al., 2019).

IEM analysis

To establish whether the observed benefits of location repetition may be brought about by preparatory biasing of visual regions representing the expected location, we used an IEM to reconstruct the target and distractor location from the pattern of EEG data. Previous research has demonstrated that these models are sensitive to the attended location during the interval preceding stimulus onset, in line with the notion that attention can prioritize target processing by sharping spatial tuning to the taskrelevant location in advance (Samaha et al., 2016; Foster et al., 2017). We thus expected target learning in our study to be associated with enhanced reconstruction of the target location before search display onset. Yet, the main question was if and how distractor learning also changes the neural representation of the distractor location in anticipation of distracting input.

Based on previous research (Samaha et al., 2016; Foster et al., 2017; van Moorselaar et al., 2018), we expected any effects to be especially present in the alpha band. An analysis collapsed across conditions and repetitions across a range of frequencies confirmed that sustained reconstruction of both the target and the distractor location was especially pronounced within the alpha band (8-12 Hz; Fig. 2). Having established that topographic distribution of alpha power tracked both the location of the target and the distractor, we examined how CTF reconstruction was modulated by location repetition within the alpha band.

\section{Target CTF}

Figure 3 shows the reconstruction of the target location in baseline and target-repeat blocks for the first and final trial in a fourtrial sequence using either evoked (phase-locked) (Fig. $3 A$ ) or total (Fig. 3B) alpha power. Target location repetitions increased spatial tuning immediately following search display onset both within evoked and total power. Reconstructions were most pronounced using evoked power, demonstrating that spatially selective activity was best captured by evoked activity, at least following target presentation. Within evoked power, this increased tuning was captured by a significant cluster that remained reliable after controlling for any differences on the first trial of the sequence (Fig. 3A, right, blue-green and gray bars). Consistent with previous findings (Samaha et al., 2016; Foster et al., 2017), only total alpha power enabled reconstruction of the target location in anticipation of display onset, confirming that target location learning sharpened spatial tuning to the target location at the neural level in advance (Fig. 3B, right, green bar). 


\section{A Evoked power}
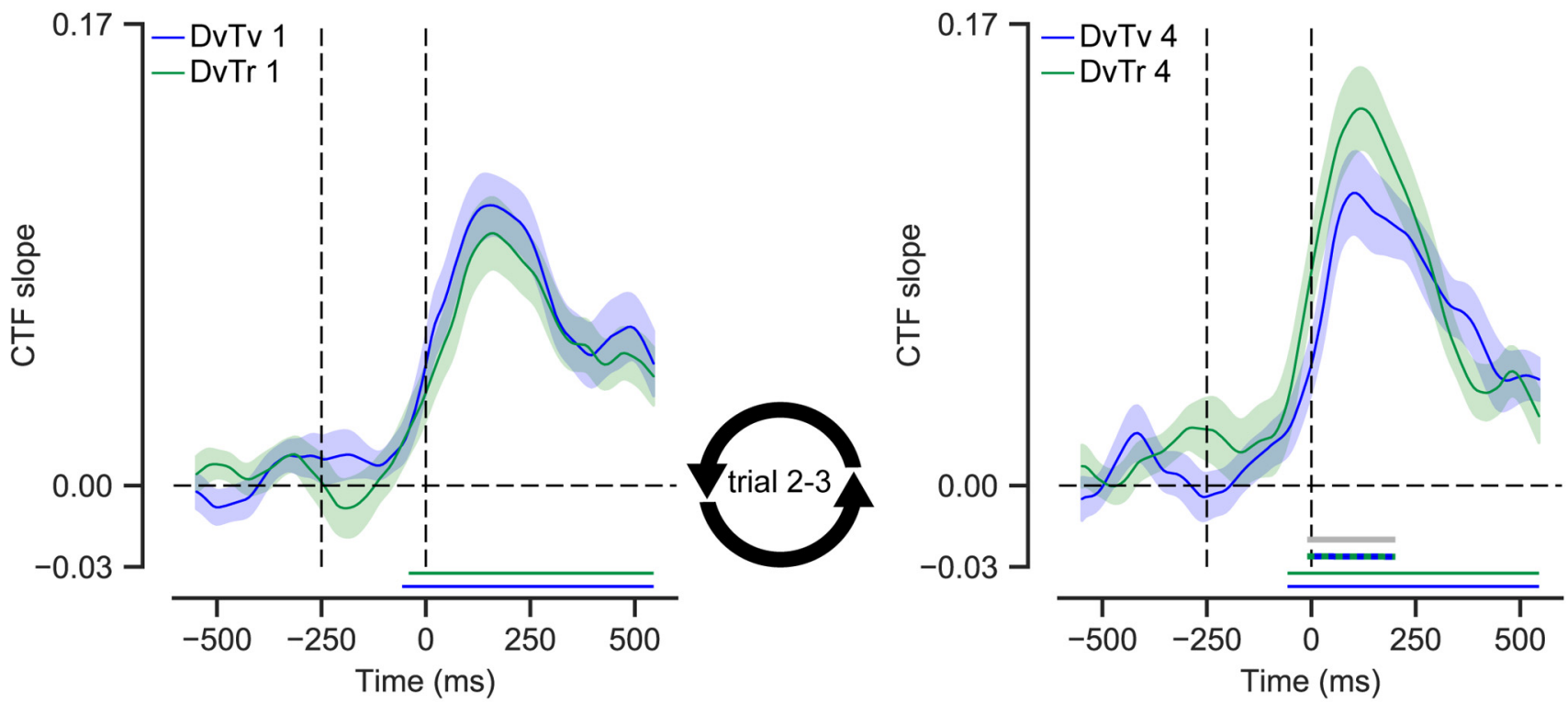

\section{B Total power}
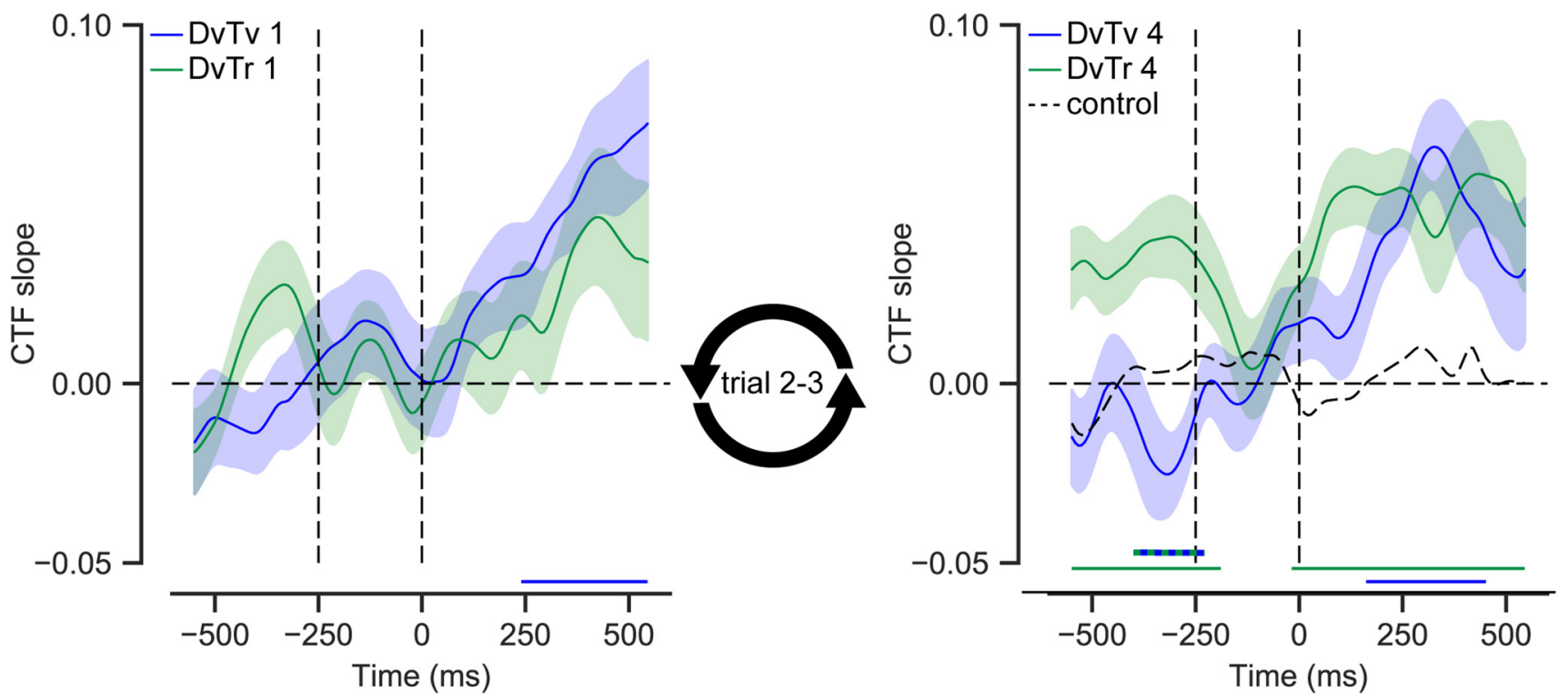

Figure 3. Target repetition increased anticipatory and poststimulus spatial tuning to target locations. All plots represent the CTF slope, which here quantifies the location specificity of the topographic distribution of activity in the alpha band. $A$, Evoked power CTF slopes tuned to the target location at the first (left) and final (right) trial in baseline (DvTv) and target-repeat sequences (DvTr). B, Total power CTF slopes tuned to the target location at the first (left) and final (right) trial in baseline and target-repeat sequences. Target repetition increased spatial tuning to the predictable target location already in advance of target presentation. A control (dotted black line) analysis showed that this effect cannot be attributed to lingering effects from the preceding trial. Shaded error bars represent bootstrapped SEM (same applies to subsequent figures). Colored bars on the $x$ axis (blue; green) represent time points where conditions differ significantly from 0 after cluster correction $(p<0.05)$. Double-colored thick lines indicate time points with a significant difference between the respective conditions after cluster correction $(p<0.05)$.

This anticipatory effect became weaker and statistically insignificant after the onset of the (empty) placeholders, suggesting that their onset may have temporarily obscured the anticipatory tuning. Although a significant cluster showed that the observed significant target repetition-related anticipatory tuning was reliably larger at the final trial relative to baseline (blue-green bar), the identified cluster was unreliable after controlling for condition differences at the first trial. We therefore explored this anticipatory tuning further using a repeated-measures ANOVA on the average tuning slopes within the prestimulus period ( -550 to 0 $\mathrm{ms})$, which critically yielded a significant interaction between condition (baseline, target repeat) and trial position $(1,4)\left(F_{(1,23)}\right.$ $\left.=4.5, p=0.045, \eta^{2}=0.16\right)$.

To exclude the possibility that lingering target processing from the preceding trial may have contributed to the observed increase in anticipatory tuning in target-repeat blocks, in a control analysis, we replaced the location labels in the baseline condition with those from the preceding trial in the sequence. If the observed anticipatory tuning following target repetition was driven by lingering activations elicited by target selection on the 


\section{A Target tuning}
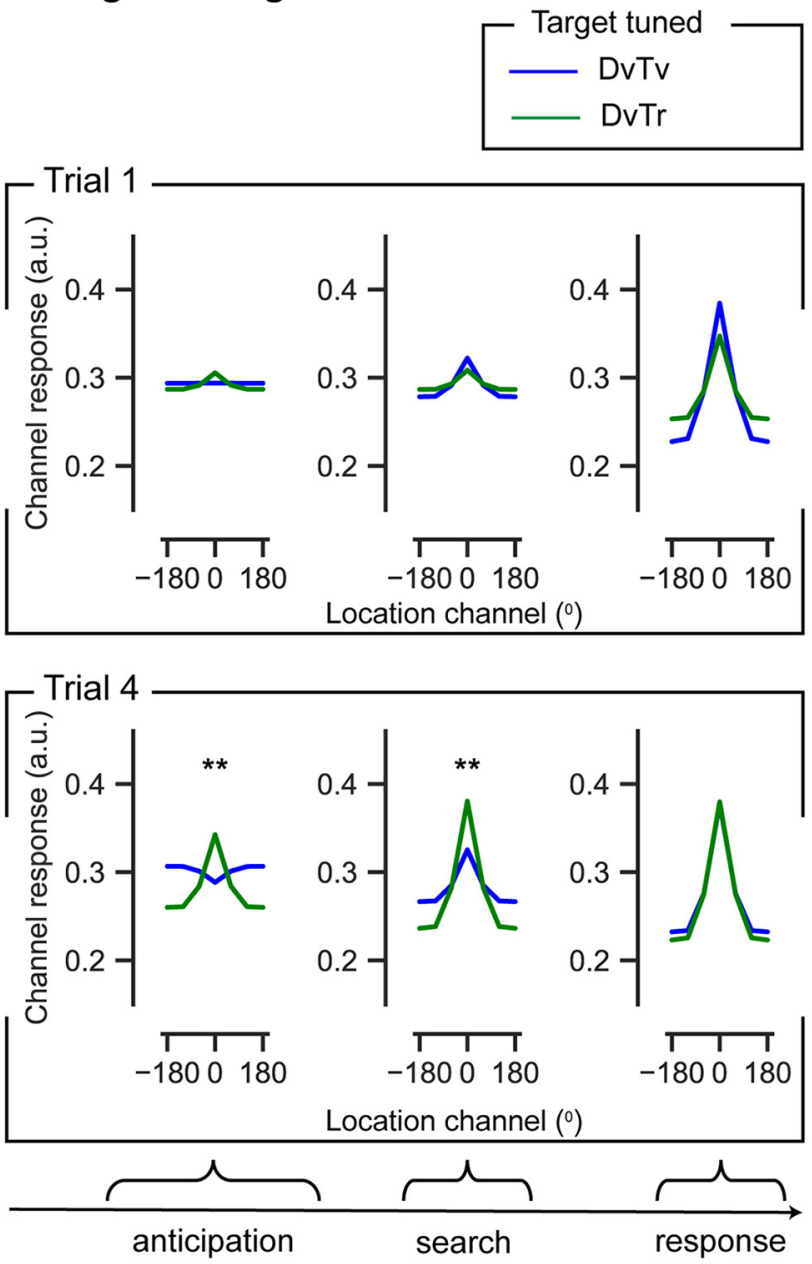

\section{B Distractor tuning}
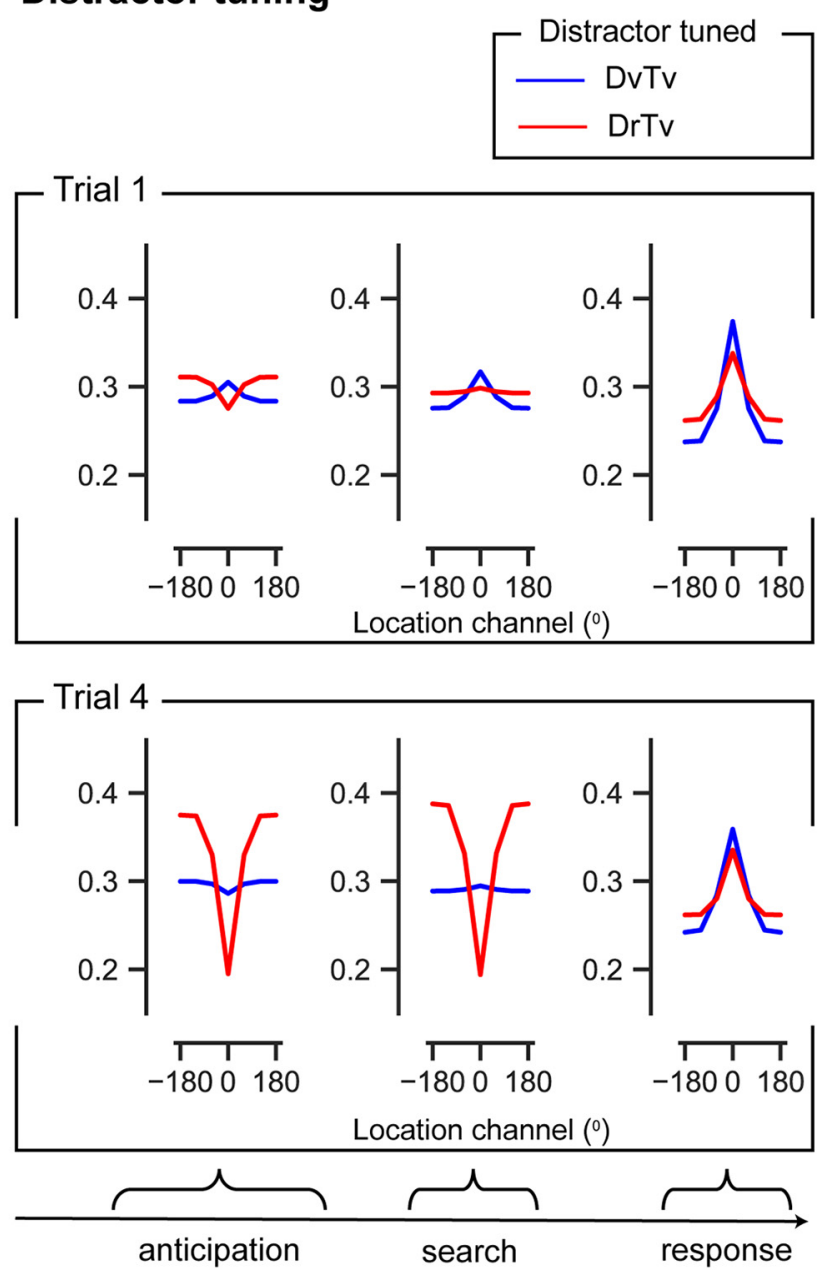

Figure 4. Estimated CTFs on the first and last trial of the sequence across three time windows of interest. $\boldsymbol{A}$, Fitted CTFs tuned to the target location in baseline (blue; DvTv) and target-repeat sequences (green; DvTr). B Fitted CTFs tuned to the distractor location in baseline (blue; DvTv) and distractor-repeat sequences (red; DrTv). Estimates were based on a fit to an exponential cosine function (for details, see Results). CTFs are shown separately for the first (top row) and final trial (bottom row) in the repetition sequence. From left to right, CTFs are averaged across three windows of interest (i.e., anticipation: -550 to $0 \mathrm{~ms}$; search display: $0-200 \mathrm{~ms}$; response: $200-550 \mathrm{~ms}$ ). ${ }^{* *} p<0.01$, significant difference between (TF amplitudes in baseline and repeat sequences. Although there was a significant difference at the final trial position for target location tuned CTF amplitudes both in anticipation and during search, no such differences were observed in distractor location tuned CTF amplitudes.

preceding trial, this analysis should also reveal anticipatory tuning. However, as shown in Figure $3 B$ (right, dotted black line), no such tuning was observed.

To better understand how learned expectations influenced target location tuning, each CTF was fitted with an exponential cosine function of the form: $\left.f(x)=\alpha\left(e^{k(\cos (\mu-x)-1}\right)\right)+\beta$, where $x$ is a vector of channel responses, $\mu, k$, and $\beta$ control the center (i.e., mean), concentration (i.e., inverse of width) and baseline (i.e., vertical offset) of the function, while $\alpha$ corresponds to the amplitude of the function (i.e., vertical scaling) (Ester et al., 2015). These fits are displayed in Figure $4 A$ across different stages of the trial. Confirming that location selectivity as captured by CTF slope is largely driven by the amplitude of the CTF, permutation tests showed that all identified condition differences based on slope were also evident in amplitude estimates. Evoked amplitudes were reliably larger between -10 and $201 \mathrm{~ms}$ both with and without controlling for any condition differences on the first trial. Also, total amplitudes reliably increased in anticipation of search display onset relative to baseline between -377 and 221 $\mathrm{ms}$ as a function of target location repetition. Interestingly, and in line with previous work (Tang et al., 2018), as visualized in Figure
$4 A$, these increases in amplitudes were accompanied by decreases in baseline estimates, resulting in significant clusters mirroring those identified by CTF slope and CTF amplitude analyses, while there was no modulation of concentration of the CTF.

Together, these results indicate that target foreknowledge was associated with enhanced tuning of spatially selective neural populations to the target location already in anticipation of the search display, and this spatial selectivity increased further in response to visual input.

\section{Distractor CTF}

We next examined how distractor learning affected the neural representation of the distractor location. Reconstruction of the distractor location, as shown in Figure 5, showed a markedly different pattern. Both within evoked (Fig. $5 A$ ) and total alpha power (Fig. $5 B$ ), we observed no postdistractor modulation of spatial tuning by distractor location repetition, nor were there reliable changes in anticipatory tuning to the distractor location. While it appears as if distractor location repetition resulted in opposite, negative tuning (see also Fig. $4 B$ ), permutation tests also identified no reliable differences in any of the estimated CTF 


\section{A Evoked power}
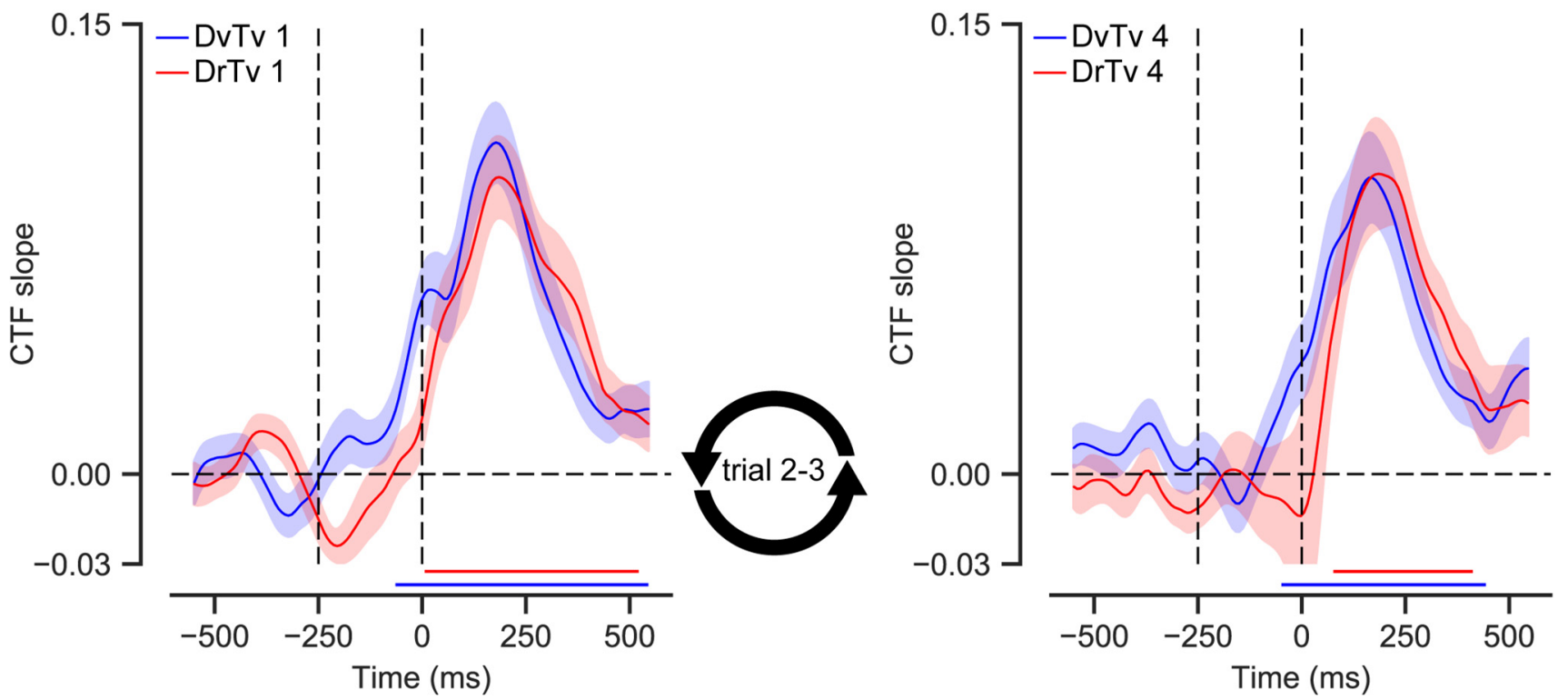

\section{B Total power}
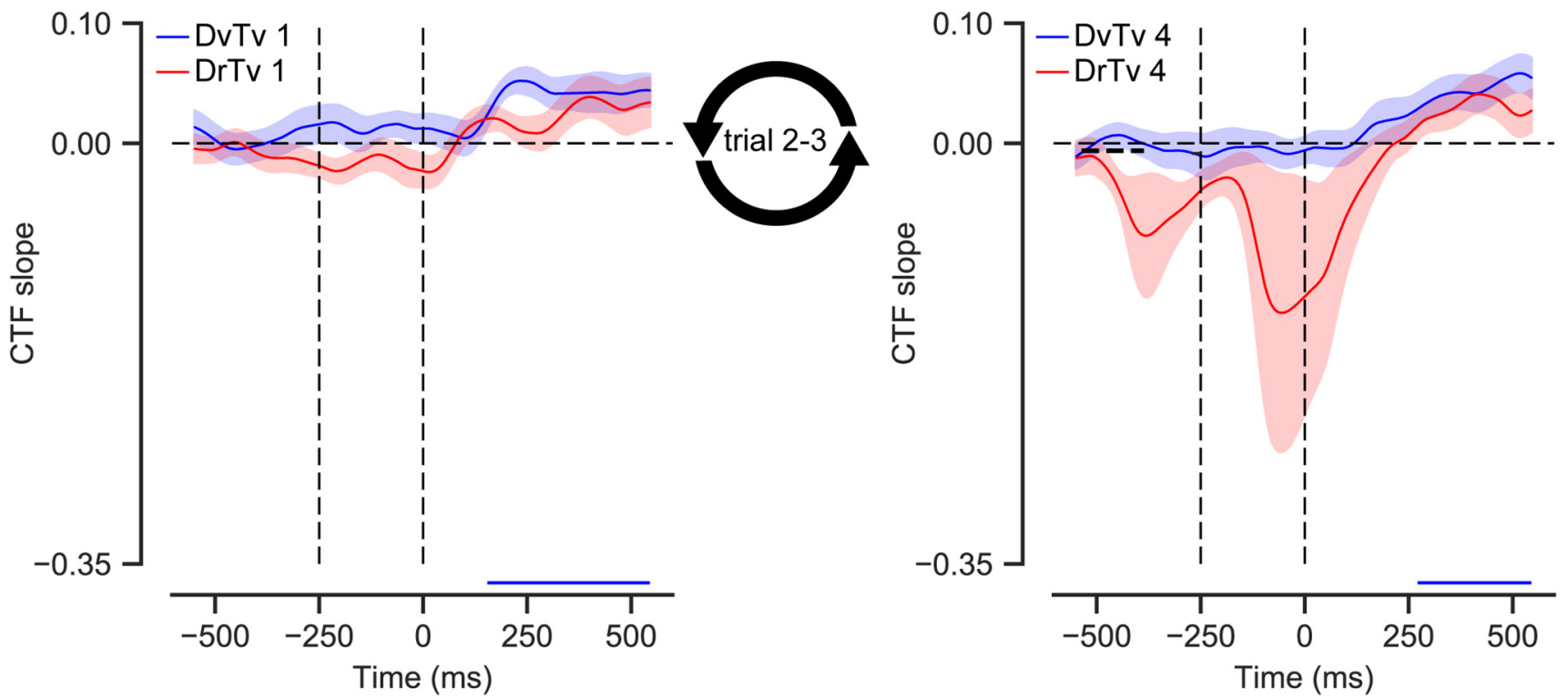

Figure 5. Distractor repetition did not change spatial tuning to distractor locations. A, Evoked alpha power (TF slopes tuned to the distractor location at the first (left) and final (right) trial in baseline (DvTv) and distractor-repeat sequences (DrTv). B, Total alpha power (TF slopes tuned to the distractor location at the first (left) and final (right) trial in baseline and distractor-repeat sequences. Colored bars on the $x$ axis (blue; red) represent time points where conditions differ significantly from 0 after cluster correction $(p<0.05)$.

parameters (i.e., amplitude, concentration, baseline) between the distractor repetition and variable conditions either. To further examine the possible anticipatory tuning, we again performed an exploratory repeated-measures ANOVA on the average tuning slopes within the prestimulus period $(-550$ to $0 \mathrm{~ms})$, which yielded no significant interaction $(F=1.2, p=0.28)$. A subsequent Bayesian analysis confirmed that the data were 2.15 less likely than equivalent models stripped of the interaction term providing anecdotal evidence against the hypothesis that distractor tuning was modulated by location repetitions (Wetzels et al., 2011). Finally, paired-sample $t$ tests contrasting amplitudes in DvTv and DrTv conditions during anticipation and immediately following search display onset were nonsignificant (all $t$ values $<1.9$, all $p$ values $>0.06$; Fig. $4 B$ ). Together, these findings provide no compelling evidence for negative tuning to the distractor location, or suggest that this effect may have been highly variable across subjects.

Thus, while target repetition was associated with both increased anticipatory and poststimulus spatial tuning, spatial tuning to the distractor location appeared unaffected by distractor repetition. This dissociation, as also visualized in Figure 4, was confirmed by a cluster-based permutation comparison between distractor-repeat and target-repeat, which yielded both an anticipatory ( $-548 \mathrm{~ms}$ to $-173 \mathrm{~ms})$ as well as a postsearch display 
A

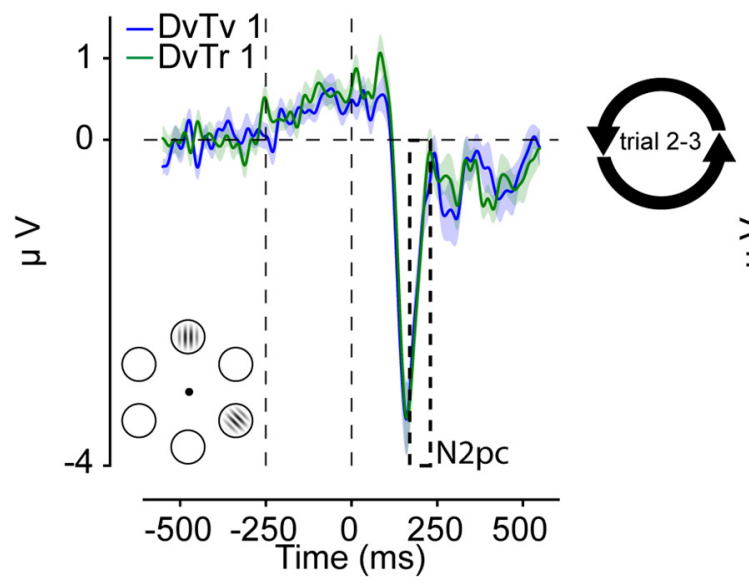

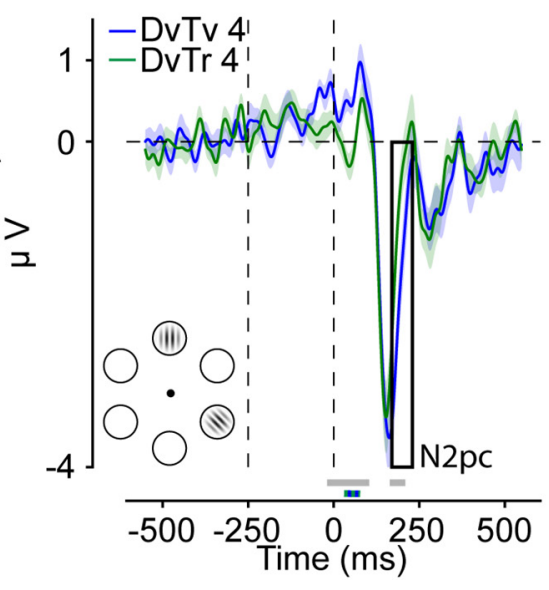

B

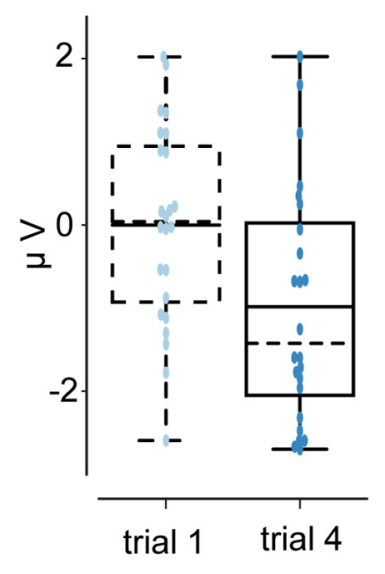

Figure 6. Target repetition reduced the amplitude of the target-evoked N2pc. ERPs evoked by targets were computed only using trials where the target was presented on the bottom left or right from fixation, with a distractor on the midline. $\boldsymbol{A}$, Difference waveforms (contralateral - ipsilateral) revealing the N2pc are shown separately for the baseline (DvTv) and target-repeat (DvTr) condition on the first (1) and final (4) repetition in the sequence. Double-colored thick lines indicate time points with a significant difference between the respective conditions after cluster correction $(p<0.05)$. Gray thick lines indicate time points with a significant condition difference after baseline correction $(p<0.05)$. B, Boxplots represent the difference between conditions (DvTv - DvTr) on the first (dashed) and final (solid) trial of a target repetition block within the N2pc window (170-230 ms). Solid lines inside boxes indicate the mean. Dashed lines indicate the median.

significant cluster $(68-278 \mathrm{~ms} ; p<0.05)$. This finding suggests that the observed reduction in distractor interference at the behavioral level is not mediated by changes in the neural representation of the distractor location, as measured with EEG and CTFs.

\section{ERP analysis}

To determine whether repetition changed early visual processing, we examined the amplitude of the visual-evoked potentials, P1 and N1. Modulations of the amplitude of these exogenous components by attention-directing spatial cues have been linked to different aspects of attention, with the P1 effect reflecting attentional inhibition and the N1 effect signaling attentional enhancement (Luck et al., 1994; Freunberger et al., 2008; Couperus and Mangun, 2010; Slagter et al., 2016). In addition, we evaluated how location learning affected later-stage target and distractor processing, as reflected in the lateralized ERP components, the $\mathrm{N} 2 \mathrm{pc}$ and the Pd, respectively. Whereas the N2pc is thought to reflect attentional selection (Luck and Hillyard, 1994; Luck, 2012; Eimer, 2014), the Pd is selectively elicited by distracting visual input and linked to distractor inhibition (Hickey et al., 2009; Gaspelin and Luck, 2018a).

\section{Target ERPs}

Contrary to our expectations, cluster-based permutation tests identified no significant effects of target repetition within the P1 or N1 time window. To further explore the effects of target repetition, mean $\mathrm{P} 1$ and $\mathrm{N} 1$ voltages were entered into separate repeated-measures ANOVAs with the within-subjects factors condition (variable vs repeat), trial position ( 1 vs 4 ), and laterality (contralateral vs ipsilateral). This analysis also revealed no condition $\times$ trial position interaction $(F=2.6, p=0.12$ for $\mathrm{P} 1 ; F=$ $3.1, p=0.09$ for $\mathrm{N} 1$ ), suggesting that early visually evoked potentials were unaffected by target repetition.

By contrast, as visualized in Figure 6, target repetition did reduce the $\mathrm{N} 2 \mathrm{pc}$, an ERP index of attentional orienting, as captured by a significant baseline-corrected cluster within the typical $\mathrm{N} 2 \mathrm{pc}$ time window. As the direct comparison on the final repetition between baseline and the target repeat condition using cluster-based permutation testing revealed no significant cluster, we also explored the $\mathrm{N} 2 \mathrm{pc}$ modulation with a repeated-measures
ANOVA with N2pc amplitude values averaged over the N2pc time window as the dependent variable. This yielded a significant three-way interaction $\left(F_{(1,23)}=8.9, p=0.007, \eta^{2}=0.28\right)$, reflecting a reduction of the $\mathrm{N} 2 \mathrm{pc}$ amplitude with target location repetition $\left(t_{(23)}=2.2, p=0.041\right)$, whereas this reduction was absent in baseline trials without repetition $(t=1.4, p=0.19)$. This set of findings again illustrates enhanced statistical power of first averaging over samples within a specific time window and conducting statistical analyses on this average versus a clusterbased permutation test on individual samples, which is conceivable more sensitive to noise. An exploratory jackknife procedure (Miller et al., 1998) demonstrated that these effects were driven by an earlier return to baseline of $\mathrm{N} 2 \mathrm{pc}$ at the final relative to the first target repetition $(\Delta=28 \mathrm{~ms}, t=3.38)$.

\section{Distractor ERPs}

ERP waveforms evoked by the distractor yielded a different pattern of results. Again, contrary to our predictions, we observed no significant effects within the $\mathrm{P} 1$ or $\mathrm{N} 1$ time windows $(F=0.0, p=$ 0.99 for $\mathrm{P} 1 ; F=0.8, p=0.37$ for N1). Like targets, distractors elicited a clear N2pc $\left(F_{(1,23)}=46.9, p<0.001, \eta^{2}=0.67\right)$, indicating that distractors captured attention on at least a subset of trials, but notably, in contrast to target repetition, this component was not modulated by distractor repetition. No significant clusters were observed within the N2pc window, nor yielded the repeated-measures ANOVA any significant interactions (all $F$ values $<2.0$, all $p$ values $>0.17$ ). At the first trial of a sequence, distractor N2pc values, however, were followed by a positivity, a positivity that was absent in target-elicited waveforms, and furthermore, given its latency and scalp topography is likely to be the $\mathrm{Pd}$, an ERP related to distractor inhibition (Gaspelin and Luck, 2018b). Interestingly, as shown in Figure 7, this positivity was greatly reduced in the final distractor repetition trial. A significant cluster obtained when contrasting baseline and distractorrepeat at the final repetition confirmed that the amplitude of this positivity reduced with distractor repetition. As the observed reduction failed to survive the cluster-based permutation baseline correction, Pd amplitudes were also evaluated via a repeatedmeasures ANOVA. A significant three-way interaction $\left(F_{(1,23)}=\right.$ 

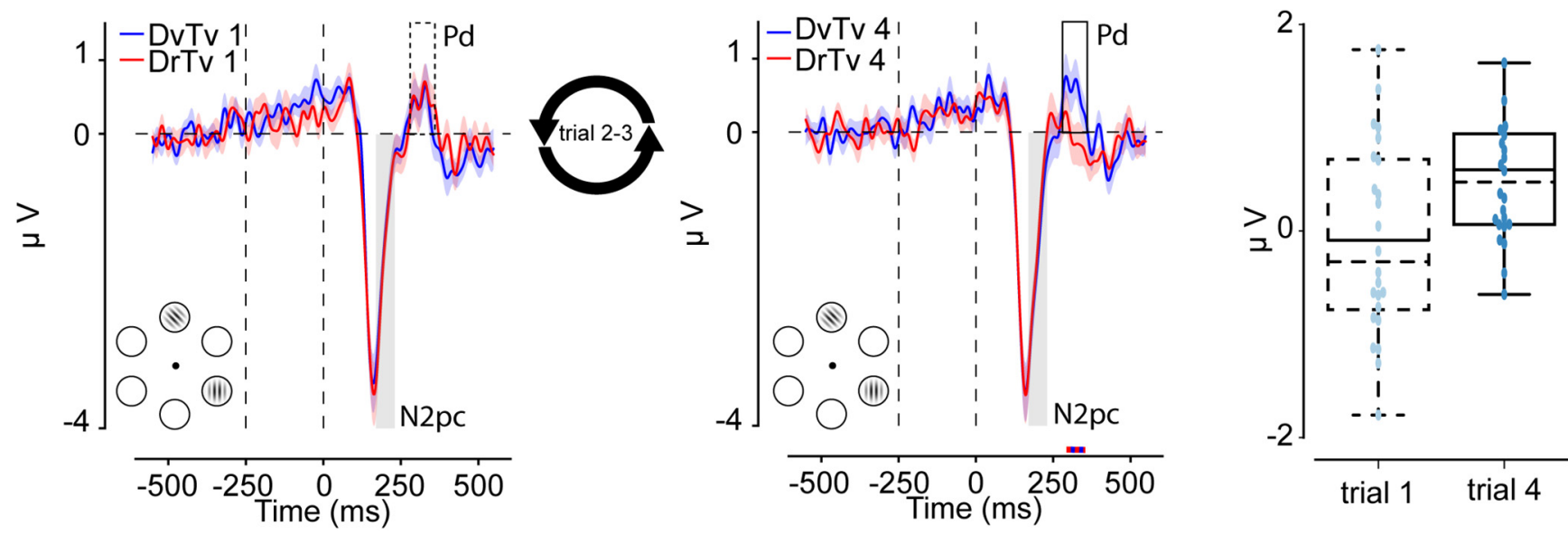

Figure 7. Distractor repetition reduced the distractor-evoked Pd. ERPs evoked by distractors were computed based on trials where the distractor was presented on the bottom left or right from fixation, with a target on the midline. $A$, Difference waveforms (contralateral - ipsilateral) revealing that the N2pc and Pd are shown separately for the baseline (DvTv) and distractor-repeat (DrTv) conditions on the first (1) and final (4) repetition in the sequence. Double-colored thick lines indicate time points with a significant difference between the respective conditions after cluster correction ( $p<0.05$ ). B, Boxplots represent the difference between conditions (DvTv - DrTv) on the first (dashed) and final (solid) repetition within the Pd window ( $280-360 \mathrm{~ms}$ ). Solid lines inside boxes indicate the mean. Dashed lines indicate the median.
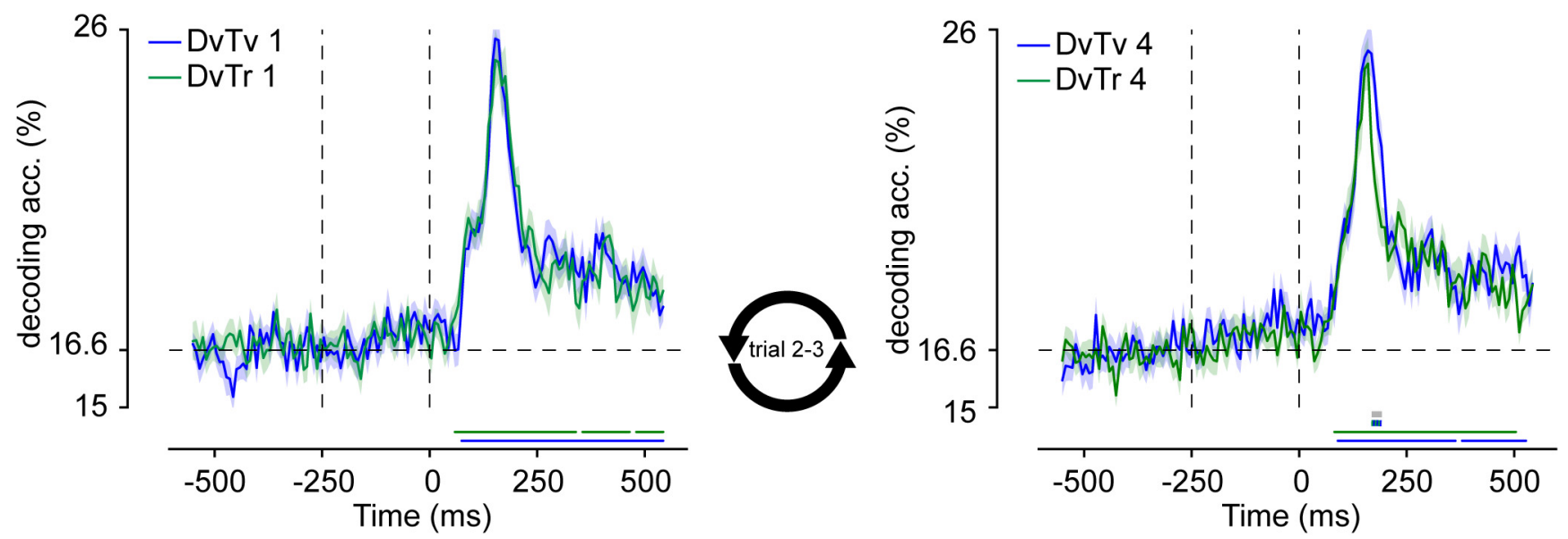

Figure 8. Target repetition was associated with a shortening of the representation of the target location within the N2pc time window, as reflected in decoding accuracy. Shown are targetlocation decoding accuracies of broadband EEG using all 64 electrodes separately for baseline (DvTv) and target-repeat (DvTr) sequences and the first (1) and last (4) trial in a sequence. Colored bars on the $x$ axis (blue; green) represent time points where conditions differ significantly from 0 after cluster correction $(p<0.05)$. Double-colored thick lines indicate time points with a significant difference between the respective conditions after cluster correction $(p<0.05)$. Gray thick lines indicate time points with a significant condition difference after baseline correction $(p<0.05)$.

$\left.4.8, p=0.038, \eta^{2}=0.17\right)$ and post hoc paired $t$ tests confirmed that the reduction of the Pd amplitude was specific to distractor repetition condition $\left(t_{(23)}=2.9, p=0.007\right)$, whereas it was absent in the baseline condition $(t=0.78,=0.44)$.

Thus, ERP analyses showed that target repetition resulted in an earlier offset of the N2pc, an ERP component associated with attentional selection. A post hoc repeated-measures ANOVA directly contrasting target and distractor $\mathrm{N} 2 \mathrm{pc}$ values at the first and final trial position yielded a marginally significant interaction $\left(F_{(1,23)}=4.0, p=0.059, \eta^{2}=0.15\right)$, demonstrating that the $\mathrm{N} 2 \mathrm{pc}$ reduction was larger in the target repeat compared with the distractor repeat condition. Whereas N2pc values elicited by targets and distractors did not differ on the first trial in the sequence $(t=0.2, p=0.83)$, after four location repetitions, the target $\mathrm{N} 2 \mathrm{pc}$ was significantly smaller than the distractor N2pc $\left(t_{(24)}=2.2, p\right.$ $<0.041)$. By contrast, distractor repetition selectively modulated the amplitude of the Pd, an ERP component associated with inhibition of distracting visual input. Importantly, this component was absent in target-elicited waveforms. Together, these findings provide additional evidence that target facilitation and distractor suppression rely on distinct neural mechanisms.

Against our predictions, however, we observed no evidence that early visual processing, as reflected in the $\mathrm{P} 1$ or the $\mathrm{N} 1$, was modulated by target or distractor location foreknowledge. Possibly, overlapping P1/N1 ERPs evoked by the other stimuli in the display masked effects by location foreknowledge on these early visual-evoked ERPs.

\section{Multivariate decoding: target location}

The classification accuracy results in Figure 8 show that the decoding model could discriminate the target location, but in contrast to IEM, only after search display onset. Moreover, target repetition reliably modulated decoding classification accuracy. Specifically, mimicking the N2pc findings, the classification peak narrowed within the N2pc time window, as reflected by a significant baseline-corrected cluster (Fig. 8). That is, decoding accuracy more quickly reduced after target learning, suggesting that target repetition may have reduced the duration of time the target 

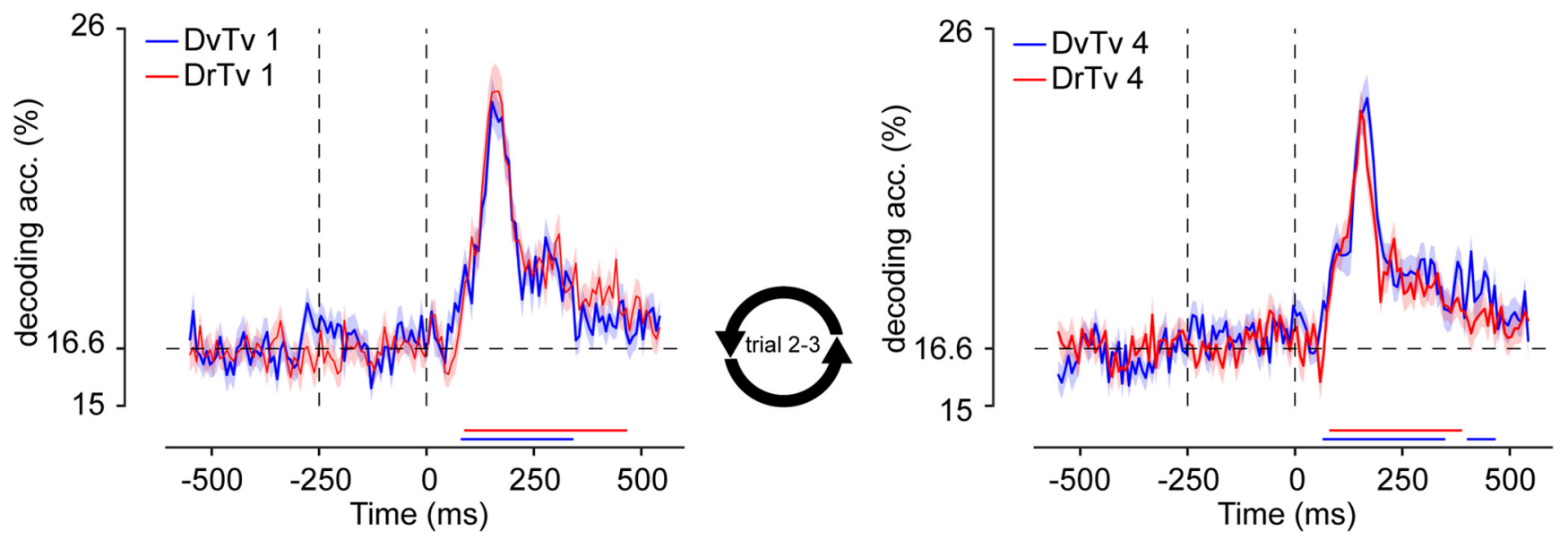

Figure 9. Distractor repetition was not associated with a change in the distractor representation, as reflected in decoding accuracy. Shown are distractor-location decoding accuracies of broadband EEG using all 64 electrodes separately for baseline (DvTv) and distractor-repeat (DrTv) sequences and the first and last trial in a sequence. Colored bars on the $x$ axis (blue; red) represent time points where conditions differ significantly from 0 after cluster correction $(p<0.05)$.

location was represented in this time window. In contrast to our IEM results, decoding did not provide evidence for target learning-induced changes in the spatial representation of the target location before target presentation. To exclude the possibility that anticipatory decoding was obscured by the broadband of frequencies used, we repeated the same analysis with alpha band filtered data (as in our IEM analysis) using either all or a set of 32 posterior electrodes. However, this exploratory analysis also only revealed poststimulus modulation of target decoding by repetition, but no anticipatory effects. Thus, the decoding results more closely followed the univariate ERP analysis results.

\section{Multivariate decoding: distractor location}

We again observed a different pattern for distractor location decoding than target location decoding. Broadband EEG decoding was also sensitive to the distractor location (Fig. 9), and only after distractor presentation, but distractor expectations did not appear to modulate classification accuracy. If anything, the classification peak within the N2pc window appeared to narrow, but this was not confirmed by cluster-based permutation tests. However, an exploratory repeated-measures ANOVA yielded a significant interaction between condition and trial position $\left(F_{(1,23)}=\right.$ 12.6, $\left.p=0.002, \eta^{2}=0.35\right)$, indicating that distractor location representation may have actually been modulated by distractor location repetition in the time window of the N2pc, as was also observed for the target location as a function of target location repetition.

\section{General Discussion}

The aim of the present study was to gain more insight into how the brain learns to ignore distracting information based on past experience, and to what extent these mechanisms differ from learning about relevant aspects of the environment. The benefits of distractor learning, which developed at a slower rate than target learning, could not be explained by more attention to nondistractor locations or by priming alone. In line with recent findings (Samaha et al., 2016; Foster et al., 2017) and in support of topdown facilitation, we found that expectations about the upcoming target location sharpened neural tuning to that location before target presentation, and furthermore, reduced post-target processes related to attentional target selection (Luck, 2012; Eimer, 2014), as indicated by the N2pc ERP component. In contrast, expectations about the upcoming distractor location only modulated postdistractor processing (i.e., reactively). Most notably, expected distractors appeared to no longer elicit a Pd, an ERP component related to distractor inhibition (Hickey et al., 2009; Gaspelin and Luck, 2018b), as if distractors were no longer considered distractors by the brain. Together, these findings suggest that target facilitation and distractor suppression are differently influenced by learning, and thus, at least in part, rely on different neural mechanisms. More generally, our results demonstrate the complementary nature of findings obtained with forward encoding modeling, univariate ERP analysis, and multivariate decoding, as these different types of analyses were in part sensitive to different aspects of the data, as discussed in more detail below.

Spatial tuning to the expected target location within the alpha band already emerged in the interval preceding visual search, likely reflecting greater top-down attention to that location (Samaha et al., 2016; Foster et al., 2017). Strikingly, no changes in spatial tuning were observed to expected distractor locations, despite clear behavioral benefits of distractor location foreknowledge and a substantial reduction in the need for reactive distractor inhibition, as captured by a much smaller distractor-evoked $\mathrm{Pd}$. These findings corroborate findings from a recent study by Noonan et al. (2016) that did not observe evidence for a role of alpha oscillations in preparatory distractor suppression, and also used a visual search task with $>2$ locations, preventing participants from simply directing more attention to the target location. Together, these findings call the computational role of alpha oscillations into question (Foster and Awh, 2018). One dominant view is that activity in sensory regions representing the distracting information can be top-down inhibited in advance through increasing alpha band activity (Jensen and Mazaheri, 2010; Foxe and Snyder, 2011), just like knowledge about upcoming targets can be used to increase the excitability of task-relevant sensory regions to prioritize target processing by releasing inhibition by alpha oscillations. Our findings and the Noonan et al. (2016) findings do not provide support for the notion that distractor inhibition is implemented through preparatory inhibition of activity in visual regions representing the distractor location. While one should always be careful when interpreting a null finding, our results are more in line with a predictive coding framework in which processing of any expected stimulus is suppressed because it provides little new information (Noonan et al., 2018). Specifi- 
cally, we found that learning about the upcoming distractor location greatly reduced the Pd component, which was selectively elicited by distractors before learning and is considered a neural marker of distractor inhibition (Hickey et al., 2009; Gaspelin and Luck, 2018b). Previous research has associated faster RTs with larger Pd amplitudes (Gaspar and McDonald, 2014) and shown that salient distractors that fail to capture attention elicit a Pd, indicative of active inhibition (Gaspelin and Luck, 2018a). Yet, here we found that distractor learning-related improvements in performance were associated with a reduction in Pd amplitude. This finding may suggest that, when a distractor is expected, there is no more need for active inhibition because the brain has learned that it can be safely ignored.

Further supporting the idea that target facilitation and distractor suppression rely on different neural mechanisms, we observed that, in contrast to distractor repetition, the N2pc amplitude by repeated targets was selectively reduced (Praamstra, 2006). Specifically, a jackknife procedure revealed the N2pc returned to baseline $28 \mathrm{~ms}$ earlier in the last versus the first trial within a target repetition sequence. This could indicate that poststimulus attentional selection was more quickly resolved. Alternatively, in line with the idea that the N2pc signals an object individuation process (Mazza and Caramazza, 2015), target individuation may have been more efficiently resolved when the target appeared at the predicted location and therefore was more precisely represented. By contrast, the N2pc elicited by distractors appeared to be insensitive to repetition effects, indicating that distractors continued to capture attention to the same extent. Distractors may have captured attention because the target location was random and attention thus had to be guided via a feature template representation in memory (left- or right-tilted Gabor), which by design was highly similar to the distractor (horizontally or vertically oriented Gabor). Previous research has demonstrated that irrelevant distractors matching the content of memory automatically capture attention (Olivers et al., 2006), an effect that can survive (implicitly) learned spatial suppression (van Moorselaar et al., 2019). It should be noted, however, that there was a numerical trend toward reduced distractor-induced activity in the time window of the N2pc in the N2pc and decoding analyses (for a similar finding, see Noonan et al., 2016). Thus, albeit to a lesser extent, distractor repetition may have also affected attentional selection or object individuation. Nonetheless, distractor learning especially modulated distractor-specific processes, as reflected by the repetition-related reduction in the Pd.

Neither target nor distraction location learning modulated early visual stimulus processing, as reflected in the amplitude of the P1 and N1. It is possible that these modulations were masked by overlapping P1/N1 ERPs evoked by the other stimuli in the display. Alternatively, and in line with the longer-latency N2pc and Pd effects, findings from several recent studies also indicate that expectations may modulate only later stages of information processing (Rungratsameetaweemana et al., 2018; Alilović et al., 2019). Thus, effects of expectation seem to strongly depend on the extent to which the predicted features are relevant for performance, whether positively (as is the case for targets) or negatively (as is the case for distractors). Once the brain has learned that the distractor stimuli can be safely ignored, they no longer need to be inhibited.

Following our ERP findings, target decoding reduced as a function of target repetition in the time window of the N2pc. Reduced decoding does not necessarily reflect a change in pattern of neural activity (i.e., location representation), as it could simply be driven by an overall signal attenuation of evoked responses.
The correspondence between the latency of our decoding and ERP findings is suggestive of this latter possibility. Distractor decoding did not reveal robust changes as a function of distractor repetition. Yet, we cannot rule out the possibility that, with more repetitions, we would have also observed a change in distractor location decoding. Four repetitions may simply not have been insufficient for observing reliable effects.

How does the brain learn that a distractor can be safely ignored? Albeit speculative, rather than modulating activity in regions representing the distractor location, distractor learning may change synaptic efficiency within these regions, analogous to long-term memory and activity-silent coding in working memory (Stokes, 2015). Synaptic memory traces provide a more efficient coding scheme than active suppression through inhibition, and could explain longer-lasting effects of learning on how attention is deployed. It would be very inefficient if the brain would need to continually actively suppress responses to irrelevant stimuli that it has learned are safe to ignore. Further research is necessary to test the possibility of distractor learning-related changes in synaptic efficiency.

As a final note, here we interpreted the output of the IEM to reflect spatial tuning of population activity. Recently, however, it has been demonstrated that CTFs do not necessarily assay population tuning as it simply recapitulates the model assumptions (i.e., the set of basis functions) rather than being an intrinsic property of the data (Gardner and Liu, 2019). In other words, if the underlying model is inaccurate, this will result in misleading parameters. Here, we point out that proactive tuning to target locations only emerged once the target location had been repeated and could thus be anticipated, and was absent in all other conditions without such foreknowledge. This finding strengthens our assumption that the underlying basis functions used by us and others (Samaha et al., 2016; Foster et al., 2017; van Moorselaar et al., 2018) form a good set of predictors of cortical tuning to spatial locations.

In conclusion, we show that, in the context of learning, distractor suppression and target facilitation rely on distinct neural mechanisms. Whereas target learning was associated with increased anticipatory spatial tuning in the alpha band, distractor learning only reduced reactive inhibition, as reflected in the amplitude of the Pd. These findings argue against direct top-down distractor inhibition, and instead resonate with predictive processing notions in which processing of expected stimuli is suppressed.

\section{References}

Alilović J, Timmermans B, Reteig LC, van Gaal S, Slagter HA (2019) No evidence that predictions and attention modulate the first feedforward sweep of cortical information processing. Cereb Cortex 29:2261-2278.

Bar M, Kassam KS, Ghuman AS, Boshyan J, Schmid AM, Dale AM, Hämäläinen MS, Marinkovic K, Schacter DL, Rosen BR, Halgren E (2006) Topdown facilitation of visual recognition. Proc Natl Acad Sci U S A 103: 449-454.

Battistoni E, Stein T, Peelen MV (2017) Preparatory attention in visual cortex. Ann N Y Acad Sci 1396:92-107.

Brouwer GJ, Heeger DJ (2009) Decoding and reconstructing color from responses in human visual cortex. J Neurosci 29:13992-14003.

Couperus JW, Mangun GR (2010) Signal enhancement and suppression during visual-spatial selective attention. Brain Res 1359:155-177.

de Vries IE, van Driel J, Olivers CN (2017) Posterior alpha EEG dynamics dissociate current from future goals in working memory guided visual search. J Neurosci 2945-2916.

Eimer M (2014) The neural basis of attentional control in visual search. Trends Cogn Sci 18:526-535.

Ester EF, Sprague TC, Serences JT (2015) Parietal and frontal cortex encode 
stimulus-specific mnemonic representations during visual working memory. Neuron 87:893-905.

Fahrenfort JJ, Grubert A, Olivers CN, Eimer M (2017) Multivariate EEG analyses support high-resolution tracking of feature-based attentional selection. Sci Rep 7:1886.

Failing M, Feldmann-Wüstefeld T, Wang B, Olivers C, Theeuwes J (2019) Statistical regularities induce spatial as well as feature-specific suppression. J Exp Psychol Hum Percept Perform. Advance online publication. Retrieved June 3, 2019. doi: 10.1037/xhp0000660.

Ferrante O, Patacca A, Di Caro V, Della Libera C, Santandrea E, Chelazzi L (2018) Altering spatial priority maps via statistical learning of target selection and distractor filtering. Cortex 102:67-95.

Fiser A, Mahringer D, Oyibo HK, Petersen AV, Leinweber M, Keller GB (2016) Experience-dependent spatial expectations in mouse visual cortex. Nat Neurosci 19:1658-1664.

Foster JJ, Sutterer DW, Serences JT, Vogel EK, Awh E (2017) Alpha band oscillations enable spatially and temporally resolved tracking of covert spatial attention. Psychol Sci 28:929-941.

Foster JJ, Awh E (2018) The role of alpha oscillations in spatial attention: limited evidence for a suppression account. Curr Opin Psychol 29:34-40.

Foxe JJ, Snyder AC (2011) The role of alpha band brain oscillations as a sensory suppression mechanism during selective attention. Front Psychol 2:154

Freunberger R, Höller Y, Griesmayr B, Gruber W, Sauseng P, Klimesch W (2008) Functional similarities between the P1 component and alpha oscillations. Eur J Neurosci 27:2330-2340.

Friston K (2009) The free-energy principle: a rough guide to the brain? Trends Cogn Sci 13:293-301.

Gardner JL, Liu T (2019) Inverted encoding models reconstruct an arbitrary model response, not the stimulus. eNeuro 6:2.

Gaspar JM, McDonald JJ (2014) Suppression of salient objects prevents distraction in visual search. J Neurosci 34:5658-5666.

Gaspelin N, Luck SJ (2018a) Combined electrophysiological and behavioral evidence for the suppression of salient distractors. J Cogn Neurosci 30:1265-1280.

Gaspelin N, Luck SJ (2018b) The role of inhibition in avoiding distraction by salient stimuli. Trends Cogn Sci 22:79-92.

Gaspelin N, Leonard CJ, Luck SJ (2015) Direct evidence for active suppression of salient-but-irrelevant sensory inputs. Psychol Sci 26:1740-1750.

Geng JJ, Behrmann M (2002) Probability cuing of target location facilitates visual search implicitly in normal participants and patients with hemispatial neglect. Psychol Sci 13:520-525.

Geng JJ, Behrmann M (2005) Spatial probability as an attentional cue in visual search. Percept Psychophys 67:1252-1268.

Gramfort A, Luessi M, Larson E, Engemann DA, Strohmeier D, Brodbeck C, Parkkonen L, Hämäläinen MS (2014) MNE software for processing MEG and EEG data. Neuroimage 86:446-460.

Hickey C, Di Lollo V, McDonald JJ (2009) Electrophysiological indices of target and distractor processing in visual search. J Cogn Neurosci 21: $760-775$.

JASP-TEAM (2018) JASP, version 0.8.2 [Computer software].

Jensen O, Mazaheri A (2010) Shaping functional architecture by oscillatory alpha activity: gating by inhibition. Front Hum Neurosci 4:186.

Jiang YV (2018) Habitual versus goal-driven attention. Cortex 102:107120.

Kok P, Mostert P, de Lange FP (2017) Prior expectations induce prestimulus sensory templates. Proc Natl Acad Sci U S A 114:10473-10478.

Luck SJ (2012) Electrophysiological correlates of the focusing of attention within complex visual scenes: N2pc and related ERP components. In: The Oxford handbook of event-related potential components, pp 329-360. Oxford: Oxford UP.

Luck SJ, Hillyard SA (1994) Electrophysiological correlates of feature analysis during visual search. Psychophysiology 31:291-308.

Luck SJ, Hillyard SA, Mouloua M, Woldorff MG, Clark VP, Hawkins HL (1994) Effects of spatial cuing on luminance detectability: psychophysical and electrophysiological evidence for early selection. J Exp Psychol Hum Percept Perform 20:887-904.

Maris E, Oostenveld R (2007) Nonparametric statistical testing of EEG-and MEG-data. J Neurosci Methods 164:177-190.

Mathôt S, Schreij D, Theeuwes J (2012) OpenSesame: an open-source, graphical experiment builder for the social sciences. Behav Res Methods 44:314-324.
Mazza V, Caramazza A (2015) Multiple object individuation and subitizing in enumeration: a view from electrophysiology. Front Hum Neurosci 9:162.

Miller J, Patterson T, Ulrich R (1998) Jackknife-based method for measuring LRP onset latency differences. Psychophysiology 35:99-115.

Myers NE, Rohenkohl G, Wyart V, Woolrich MW, Nobre AC, Stokes MG (2015) Testing sensory evidence against mnemonic templates. Elife 4:e09000.

Noonan MP, Adamian N, Pike A, Printzlau F, Crittenden BM, Stokes MG (2016) Distinct mechanisms for distractor suppression and target facilitation. J Neurosci 36:1797-1807.

Noonan MP, Crittenden BM, Jensen O, Stokes MG (2018) Selective inhibition of distracting input. Behav Brain Res 355:36-47.

Nyström M, Holmqvist K (2010) An adaptive algorithm for fixation, saccade, and glissade detection in eyetracking data. Behav Res Methods 42:188-204.

Oliphant TE (2007) Python for scientific computing. Comput Sci Eng 9:10-20.

Olivers CN, Meijer F, Theeuwes J (2006) Feature-based memory-driven attentional capture: visual working memory content affects visual attention. J Exp Psychol Hum Percept Perform 32:1243-1265.

Oostenveld R, Fries P, Maris E, Schoffelen JM (2011) FieldTrip: open source software for advanced analysis of MEG, EEG, and invasive electrophysiological data. Comput Intell Neurosci 2011:156869.

Pedregosa F, Varoquaux G, Gramfort A, Michel V, Thirion B, Grisel O, Dubourg V (2011) Scikit-learn: machine learning in Python. J Machine Learn Res 12:2825-2830.

Peirce JW (2009) Generating stimuli for neuroscience using PsychoPy. Front Neurosci 2:10.

Perrin F, Pernier J, Bertrand O, Echallier JF (1989) Spherical splines for scalp potential and current density mapping. Electroencephalogr Clin Neurophysiol 72:184-187.

Praamstra P (2006) Prior information of stimulus location: effects on ERP measures of visual selection and response selection. Brain Res 1072: 153-160.

Rao RP (2005) Bayesian inference and attentional modulation in the visual cortex. Neuroreport 16:1843-1848.

Rao RP, Ballard DH (1999) Predictive coding in the visual cortex: a functional interpretation of some extra-classical receptive-field effects. Nat Neurosci 2:79-87.

Rungratsameetaweemana N, Itthipuripat S, Salazar A, Serences JT (2018) Expectations do not alter early sensory processing during perceptual decision making. J Neurosci 38:5632-5648.

Samaha J, Sprague TC, Postle BR (2016) Decoding and reconstructing the focus of spatial attention from the topography of alpha band oscillations. J Cogn Neurosci 28:1090-1097.

Slagter HA, Prinssen S, Reteig LC, Mazaheri A (2016) Facilitation and inhibition in attention: functional dissociation of prestimulus alpha activity, P1, and N1 components. Neuroimage 125:25-35.

Stokes MG (2015) 'Activity-silent' working memory in prefrontal cortex: a dynamic coding framework. Trends Cogn Sci 19:394-405.

Tang MF, Smout CA, Arabzadeh E, Mattingley JB (2018) Prediction error and repetition suppression have distinct effects on neural representations of visual information. Elife 7:e33123.

van Moorselaar D, Foster JJ, Sutterer DW, Theeuwes J, Olivers CNL, Awh E (2018) Spatially selective alpha oscillations reveal moment-by-moment trade-offs between working memory and attention. J Cogn Neurosci 30:256-266.

van Moorselaar D, Theeuwes J, Olivers CN (2019) Memory-based attentional biases survive spatial suppression driven by selection history. Visual Cognition. Advance online publication. Retrieved March 5, 2019. doi: $10.1080 / 13506285.2019 .1582571$

Wang B, Theeuwes J (2018a) How to inhibit a distractor location? Statistical learning versus active, top-down suppression. Atten Percept Psychophys $80: 860-870$.

Wang B, Theeuwes J (2018b) Statistical regularities modulate attentional capture. J Exp Psychol Hum Percept Perform 44:13-17.

Wetzels R, Matzke D, Lee MD, Rouder JN, Iverson GJ, Wagenmakers EJ (2011) Statistical evidence in experimental psychology: an empirical comparison using $855 t$ tests. Perspect Psychol Sci 6:291-298.

Woodman GF, Luck SJ (2003) Serial deployment of attention during visual search. J Exp Psychol Hum Percept Perform 29:121-138. 